\title{
Late Pleistocene Canidae remains from Geographical Society Cave in the Russian Far East
}

\author{
Gennady F. Baryshnikov
}

\begin{abstract}
The analysis of bone remains of canids from the Upper-Pleistocene deposits of Geographical Society Cave in Primorskii Territory, Russia, revealed the presence of 4 species: Nyctereutes procyonoides, Canis lupus, Cuon alpinus, and Vulpes vulpes. Their accumulation is associated predominantly with the food activity of larger carnivores (Crocuta ultima, Panthera tigris), which used the cave as a den. No reliable signs of utilization of canids by ancient people were detected.
\end{abstract}

KEY WORDS: Canidae, Late Pleistocene, Paleolithic cave sites, Russian Far East, taphonomy, taxonomy.

Gennady F. Baryshnikov [G_Baryshnikov@mail.ru], Zoological Institute, Russian Academy of Sciences, Universitetskaya nab. 1, Saint Petersburg 199034, Russia.

\section{Позднеплейстоценовые остатки псовых (Canidae) из пещеры Географического общества на Дальнем Востоке России}

\section{Г.Ф. Барышников}

РЕЗЮМЕ. Анализ костных остатков канид из верхнеплейстоценовых отложений пещеры Географического общества (Приморский край, Россия) показал присутствие 4 видов: Nyctereutes procyonoides, Canis lupus, Cuon alpinus and Vulpes vulpes. Их накопление связано преимущественно с пищевой активностью крупных хищников (Crocuta ultima, Panthera tigris), использовавших пещеру как свое логово. Нет достоверных следов утилизации канид древним человеком.

КЛЮЧЕВЫЕ СЛОВА: Canidae, поздний плейстоцен, палеолитические пещерные стоянки, Дальний Восток России, тафономия, систематика.

\section{Introduction}

Recent Canidae are known to be represented in the southern part of the Russian Far East by four species: Nyctereutes procyonoides (Gray, 1834), Canis lupus L., 1758, Cuon alpinus (Pallas, 1811), and Vulpes vulpes (L., 1758). Cuon alpinus disappeared from this region during last decades. Ovodov (1977) included these species into his preliminary list of the Late Pleistocene mammals from the Geographical Society Cave situated at Partizanskaya River (former Suchan River) near Nakhodka City in Primorskii Territory $\left(42^{\circ} 93^{\prime} \mathrm{N}\right.$, $\left.133^{\circ} 05^{\prime} \mathrm{E}\right)$.

In the present study, I re-identified and morphologically characterized all canid material from this cave for the first time. The present material is housed in the Zoological Institute of Russian Academy of Sciences in Saint Petersburg and came predominantly from 19661967 excavations by N. Ovodov and from the collection of the local history researcher E. Leshok from 1972.

Fossil large mammal remains from Geographical Society Cave are referred to hyena, wolf, mammoth, woolly rhino, horse, bison, deer, elk, goral, and other inhabitants of forest and grassy or rocky landscapes. This implies a great biological and taxonomical diver- sity of the Late Pleistocene fauna in the southern part of the Russian Far East. The cave yielded scarce stone artifacts indicating visits of ancient hominins. It is not yet known who these visitors were.

A detailed description of the cave site has been earlier provided by Baryshnikov (2014). The stone implements and significant osteological material are mainly associated with the layer 4 (Ovodov, 1977). However, a greater part of fossil records has no stratigraphical connection, being characterized only by a depth of their occurrence. Hyena bones provided six AMS ${ }^{14} \mathrm{C}$ dates, from 34510 to $48650 \mathrm{BP}$ (Kuzmin et al., 2001; Rohland et al., 2005; Stuart \& Lister, 2014), which refer a time of the formation of bone-bearing layer in Geographical Society Cave to the warm stage of the Late Pleistocene (MIS 3).

Institutional abbreviations: GMMKU - Geology-Mineralogy Museum of Kazan University, Kazan, Russia; GMY - Geological Museum, Yakutsk, Russia; ISAK - Institute of Systematics and Evolution of Animals, Krakow, Poland; NHM - Natural History Museum, London, Great Britain; NHMB - Natural History Museum, Berlin, Germany; NHMP — National Museum, Prague, Czech Republic; ZIN - Zoological Institute, Russian Academy of Sciences, St. Petersburg, Russia. 


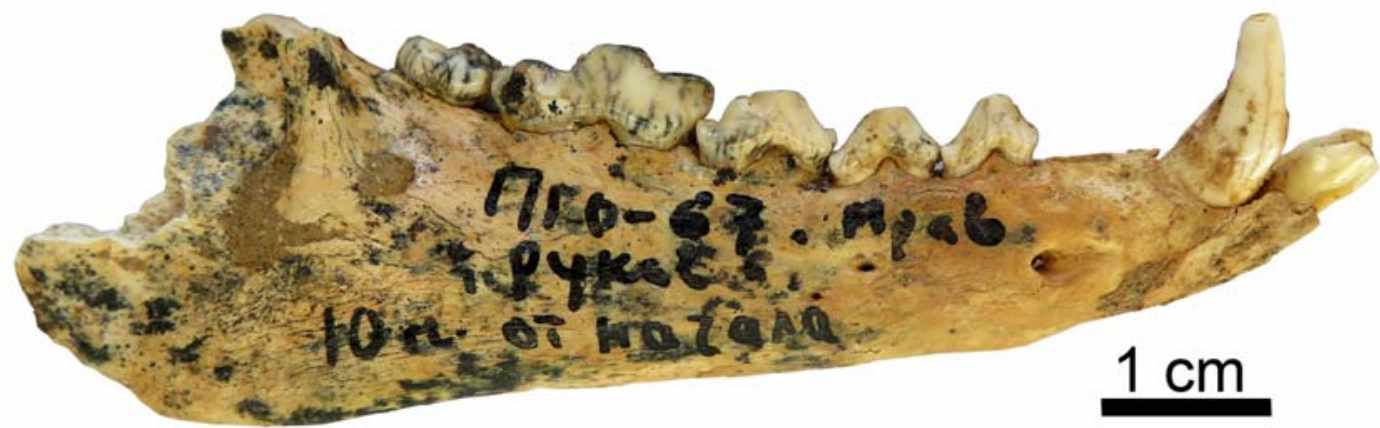

Fig. 1. Nyctereutes procyonoides, right mandible (ZIN 37290) from Geographical Society Cave, buccal view.

Measurements. Dental measurements: $L$ - greatest length, Lpa - length of paracon, Ltrd - length of talonid, $W$ - greatest width. Vertebrae measurements: $\mathrm{BF} C$ - - breadth of the caudal articular surface, $\mathrm{BFCr}$ breadth of the cranial articular surface, $B P a c d$ - breadth between processi articulares caudales, $G L$ - greatest length of atlas, $H$ - height, $L A d$ - length of the dorsal arch, median, $L A P a$ - length of the arch including the prosessus articularis caudalis, $L C D e$ - length in the region of the corpus including the dens. Limb bones measurements: $B d$ - breadth of the distal end, $B G$ breadth of the glenoid cavity, $B p$ - breadth of the proximal end, $D d$ - breadth of the distal end, $D p$ breadth of the proximal end, $D P A$ - depth across the processus anconaeus, $G B$ - greatest breath, $G L$ greatest length, GLP - greatest length of glenoid process, $L A R$ - length of the acetabulum on the rim, $S D$ breadth of the diaphysis in medium part, $S D O$ - smallest depth of the olecranon, $\mathrm{SH}$ - smallest height of the shaft of ilium, SLC - smallest length of neck of the scapula. Measurements were taken in accordance with the scheme by von den Driesch (1976).

\section{Systematic part}

Family Canidae Fisher, 1817

Genus Nyctereutes Temminck, 1838

Nyctereutes procyonoides (Gray, 1834)

Ovodov (1977) found four bones of raccoon dog in the Pleistocene deposits of Geographical Society Cave. No remains were reliably associated with the lower levels. I provisionally referred to this level the left mandible (ZIN 37290), based on the degree of its fossilization.

The specimen ZIN 37290 exhibits three incisors, a canine, alveolus of p1, and cheek teeth $\mathrm{p} 2-\mathrm{m} 2$ (Fig. 1). Alveolus of $\mathrm{m} 3$ is absent. The mandible is high and has

Table 1. Measurements (mm) of mandibles of Nyctereutes procyonoides.

\begin{tabular}{|c|c|c|c|c|}
\hline \multirow{3}{*}{ Measurements } & \multirow{3}{*}{$\begin{array}{c}\text { Late Pleistocene } \\
\text { Geographical Society Cave, Russia } \\
\text { ZIN } 37290\end{array}$} & \multicolumn{2}{|c|}{ N. p. ussuriensis, recent } & \multirow{3}{*}{$\begin{array}{c}\text { N.p. viverrinus, recent } \\
\text { Honshu, Japan } \\
\text { ZIN } 32542\end{array}$} \\
\hline & & \multicolumn{2}{|c|}{ Primorskii Territory } & \\
\hline & & ZIN 2684, $0^{7}$ & ZIN 9270 & \\
\hline $\mathrm{Lc} 1-\mathrm{m} 2$ & 52.2 & 48.3 & 51.3 & 48.6 \\
\hline Lp2-m2 & 38.9 & 36.4 & 39.2 & 36.7 \\
\hline Lp1-p4 & ca25 & 22.5 & 24.3 & 22.0 \\
\hline $\mathrm{Lm} 1-\mathrm{m} 2$ & 19.8 & 18.0 & 19.8 & 18.7 \\
\hline Height behind $\mathrm{m} 1$ & 16.9 & 13.8 & 14.4 & 12.9 \\
\hline Height behind $\mathrm{p} 2$ & 11.0 & 10.4 & 10.4 & 9.0 \\
\hline \multicolumn{5}{|l|}{ Teeth } \\
\hline Lc1 & 6.3 & 5.3 & 6.4 & 5.8 \\
\hline Lc1 & 4.3 & 3.5 & 4.0 & 3.7 \\
\hline Lp2 & 5.9 & 5.3 & 5.6 & 5.4 \\
\hline $\mathrm{Wp} 2$ & 2.6 & 2.6 & 2.8 & 2.4 \\
\hline Lp3 & 6.4 & 6.2 & 6.4 & 6.0 \\
\hline Wp3 & 2.8 & 2.8 & 3.1 & 2.5 \\
\hline Lp4 & 8.4 & 7.0 & 7.4 & 6.9 \\
\hline Wp4 & 3.6 & 3.4 & 3.9 & 3.5 \\
\hline Lm1 & 13.3 & 12.1 & 13.0 & 12.4 \\
\hline Ltldm1 & 4.0 & 3.9 & 4.4 & 4.3 \\
\hline Wm1 & 5.1 & 4.7 & 5.5 & 5.2 \\
\hline $\mathrm{Lm} 2$ & 6.5 & 5.6 & 7.2 & 6.5 \\
\hline $\mathrm{Wm} 2$ & 4.2 & 3.9 & 4.3 & 4.2 \\
\hline
\end{tabular}




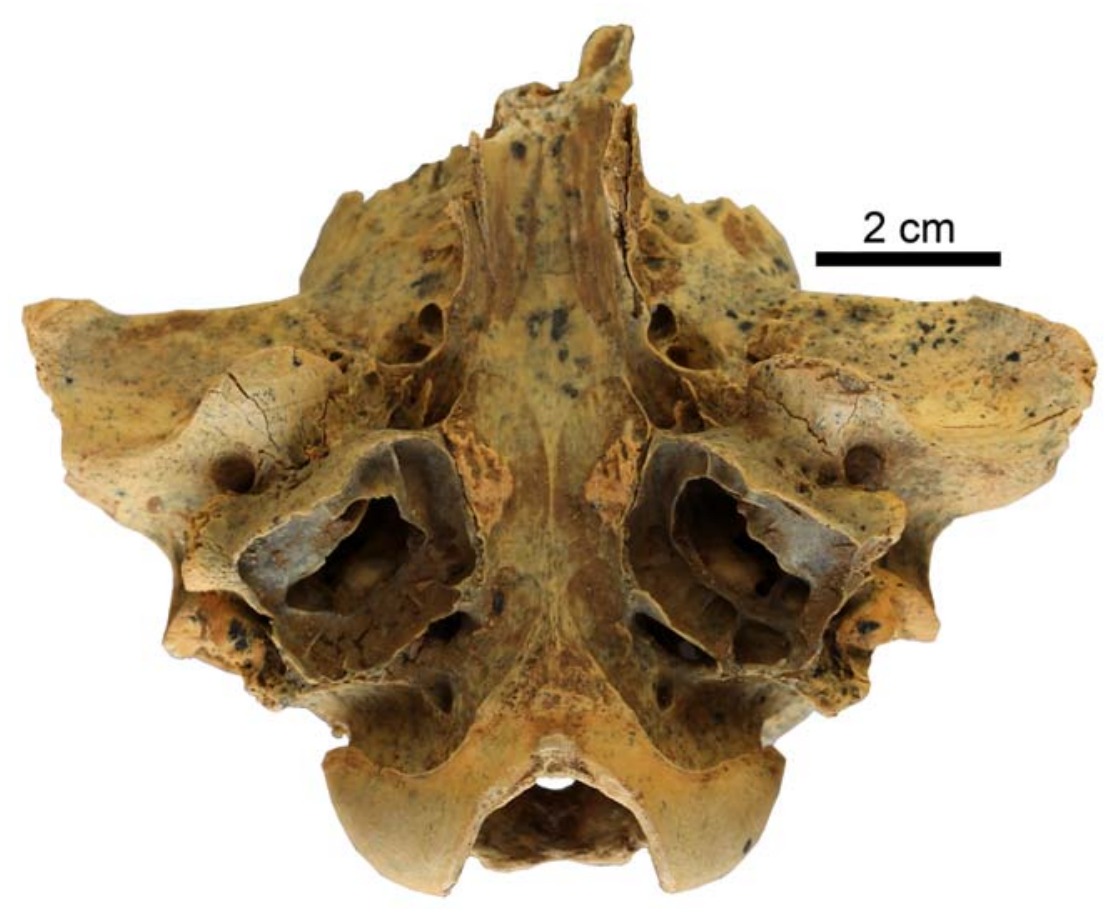

Fig. 2. Canis lupus, cranial fragment (ZIN 37265) from Geographical Society Cave, ventral view.

preangular lobe, which is characteristic of $N$. procyonoides. Cheek teeth are apically worn. By its dimensions, the fossil finding approaches to the largest specimens of the continental subspecies $N$. p. ussuriensis (Matschie, 1907), which now occurs in the southern part of the Russian Far East, and exceeds the size of mandibles of the insular subspecies $N$. p. viverrinus Temminck, 1838 from Japan (Tab. 1). Especially impressive seems to be a pronounced height of the body of mandible and a marked length of carnassial tooth. Even larger size of $\mathrm{m} 1$ was observed in the fossil $N$. cf. sinensis from Zhoukoudian (Locality 3) in China (Pei, 1936).

Among the premolars p2-p4, only p4 bears well pronounced posterior accesory cusp. The lower carnassial tooth $\mathrm{m} 1$ has a large metaconid. Talonid of this tooth is characterized by wide and deep basin divided into two parts by transverse ridges running from hypoconid and entoconid. The latter is smaller than hypoconid and is shifted farther backwards. Hypoconulid is not developed. The molar $\mathrm{m} 2$ shows a high metaconid and rather small talonid with depression in inner part.

\section{Genus Canis Linnaeus, 1758 \\ Canis lupus Linnaeus, 1758}

By the number of bone remains found in the cave, wolf occupies the first place among Carnivora. Ovodov (1977) points out 390 bones from 13 individuals. I have counted 392 wolf specimens.

Description. Skull fragments. There is the rostral part of cranium with P2 and P3 (ZIN 37274-2), which breadth in the area of canines is nearly $50 \mathrm{~mm}$, as well as the upper part of neurocranium (ZIN 37274-1) with minimal width $40.5 \mathrm{~mm}$. Ones more fragment represents the part of basicranium (ZIN 37265) with open auditory bullae and with both occipital condyles (Fig. 2 ). The breadth at occipital condyles is $48.4 \mathrm{~mm}$; the mastoid breadth is nearly $83 \mathrm{~mm}$. Inner margins of auditory bullae run non-parallel as in the recent $C$. lupus, being, however, more pronouncedly approximated to each other.

Mandibles are high. Their height behind $\mathrm{ml}$ constitutes $30.2-37.2 \mathrm{~mm}$ (mean $34.14 \mathrm{~mm}, n=5$ ), the height behind p2 varies from $26.9 \mathrm{~mm}$ to $31.8 \mathrm{~mm}$ (mean $29.14 \mathrm{~mm}, n=11$ ). By these dimensions, mandibles exceed not only the examined specimens from the Late Pleistocene of Europe (Tab. 2), but also the mandibles from the Late Pleistocene of Yakutia in Russia. In the latter, the height behind $\mathrm{ml}$ is $26.0-34.7 \mathrm{~mm}$ (mean $30.13 \mathrm{~mm}, n=7)$ and the height behind p2 is 23.3-29.7 $\mathrm{mm}$ (mean $25.97 \mathrm{~mm}, n=8$ ). Mandibles of the recent $C$. lupus from Primorskii Territory are also markedly lower, their height behind $\mathrm{m} 1$ is evaluated as 26.2-33.6 $\mathrm{mm}$ in males (mean $29.26 \mathrm{~mm}, n=8$ ) and as 23.6-27.6 $\mathrm{mm}$ in females (mean $25.75 \mathrm{~mm}, n=8$ ), whereas the height behind p2 corresponds to $21.8-25.9 \mathrm{~mm}$ in males (mean $24.12 \mathrm{~mm}, n=8$ ) and 18.6-23.9 $\mathrm{mm}$ in females (mean $21.14 \mathrm{~mm}, n=8$ ).

Upper teeth. By their shape, upper and lower incisors reveal no difference from those of the recent $C$. lupus (Fig. 3). Their dimensions vary depending on the degree of wear and, probably, on sex. The measurements of upper incisors are: $\mathrm{I} 1(\mathrm{~L}=6.8 \mathrm{~mm}, \mathrm{~W}=6.2 \mathrm{~mm}$, $n=1), \mathrm{I} 2(\mathrm{~L}=7.6,7.8,8.8,9.5 \mathrm{~mm}, \mathrm{~W}=7.0,6.1,7.8,8.7$ $\mathrm{mm}$, correspondingly; $n=4)$ and $\mathrm{I} 3(\mathrm{~L}=9.9,10.2,10.2$ $\mathrm{mm}, \mathrm{W}=7.5,8.2,8.8 \mathrm{~mm}$, correspondingly; $n=3)$. The 
Table 2. Measurements (mm) of upper tooth rows of Late Pleistocene Canis lupus.

\begin{tabular}{|c|c|c|c|c|c|c|c|}
\hline \multirow{2}{*}{$\begin{array}{l}\text { Measure- } \\
\text { ments }\end{array}$} & \multicolumn{2}{|c|}{$\begin{array}{c}\text { Geographical Society Cave, } \\
\text { Russia }\end{array}$} & \multirow{2}{*}{$\begin{array}{c}\text { Khaptashinsky Yar, } \\
\text { Yakutia, Russia } \\
\text { GMY 3728, ad. }\end{array}$} & \multicolumn{2}{|c|}{ Gailenreuth, Germany } & \multicolumn{2}{|c|}{$\begin{array}{l}\text { Srbsko Chlum-Komín } \\
\text { Cave, Czech Republic }\end{array}$} \\
\hline & $\begin{array}{l}\text { ZIN } 37267, \\
\text { sen. }\end{array}$ & $\begin{array}{l}\text { ZIN } 37266 \\
\text { ad. }\end{array}$ & & NHM 404b & NHM 403 & $\begin{array}{l}\text { NHMP } \\
\text { R3717 }\end{array}$ & $\begin{array}{l}\text { NHMP } \\
\text { R5228 }\end{array}$ \\
\hline LP4-M2 & 44.9 & 45.8 & 47.4 & 49.3 & 43.4 & 46.2 & 46.9 \\
\hline LM1-M2 & 24.4 & 25.5 & 25.8 & 27.3 & 24.0 & & \\
\hline \multicolumn{8}{|l|}{ Teeth } \\
\hline LP4 & 25.5 & 25.4 & 26.7 & 26.8 & 23.8 & 26.6 & 25.8 \\
\hline LpaP4 & 17.3 & 16.7 & & & & 17.8 & 17.2 \\
\hline WP4 & 15.0 & 13.4 & 13.4 & 16.3 & 15.0 & 15.2 & 15.5 \\
\hline LM1 & 16.2 & 15.7 & 16.2 & 17.8 & 15.9 & 16.4 & 16.7 \\
\hline WM1 & 19.8 & 18.2 & 20.5 & 21.0 & 18.0 & 19.7 & 19.7 \\
\hline LM2 & 8.2 & 7.6 & 9.5 & 9.7 & 7.9 & 8.3 & 8.8 \\
\hline WM2 & 11.0 & 10.6 & 12.9 & 12.6 & 10.7 & 11.8 & 11.5 \\
\hline
\end{tabular}
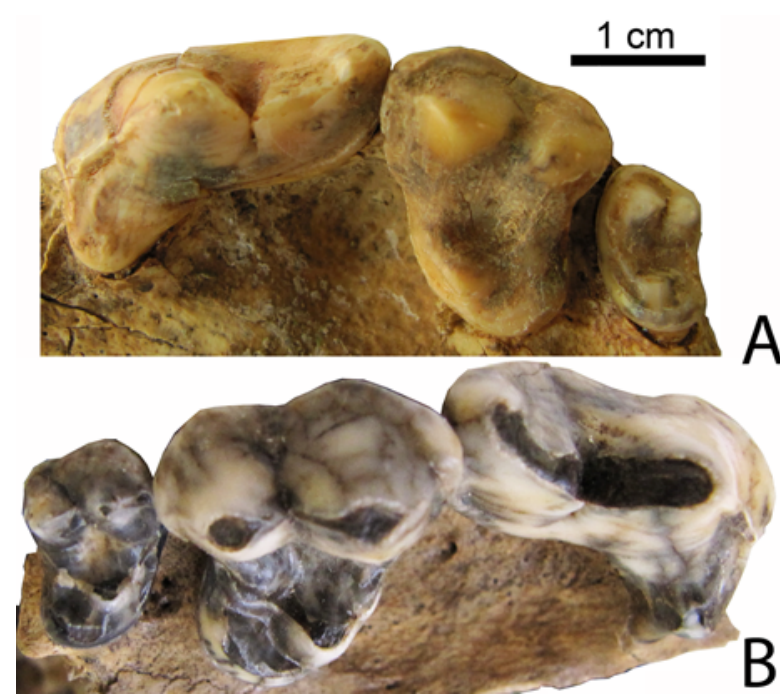

Fig. 3. Canis lupus, upper teeth row P4-M2 from Geographical Society Cave, Russia (A) and from Srbsko ChlumKomín Cave, Czech Republic (B); occlusal view.

A - ZIN 37266, left; B - NHMP R3717, right.

largest specimens exceed maximum size of incisors in the recent $C$. lupus from Primorskii Territory.

Males of C. lupus are known to be larger than females; the difference in size is well apparent in the dimensions of canines. In the sample of the recent wolf from Primorskii Territory, the anterior-posterior length of the male upper canine constitutes $12.7-14.5 \mathrm{~mm}$ and width $7.4-8.8 \mathrm{~mm}(n=9)$. In females the upper canine varies from $11.6 \mathrm{~mm}$ to $12.8 \mathrm{~mm}$ in length and from 6.8 to $8.0 \mathrm{~mm}$ in width $(n=8)$. The fossil sample contains six male canines exceeding $13.0 \mathrm{~mm}(13.1-14.9 \mathrm{~mm})$ in length and $9.0 \mathrm{~mm}(9.3-10.0 \mathrm{~mm})$ in width. Two specimens $(\mathrm{W}=8.1,8.9 \mathrm{~mm})$ are referred to female.

A single $\mathrm{P} 2(\mathrm{~L}=14.0 \mathrm{~mm}, \mathrm{~W}=6.2 \mathrm{~mm})$ bears a distinct posterior accessory cusp. P3 markedly varies in size. Maximum length of ZIN 37274-3 exceeds that of P3 in all Pleistocene wolves measured (Tab. 4). The posterior cusp is well developed; a knob-like postcin- gulum is well defined behind it.

The upper carnassial tooth $\mathrm{P} 4$ is typical of C. lupus. The greatest length $(25.4-28.0 \mathrm{~mm}, n=3)$ somewhat surpasses this dimension in the recent wolf from Primorskii Territory $(22.1-26.1 \mathrm{~mm}, n=20)$. The protocone is slender and is located far ahead of the level of the paracone apex. The length of metastylar blade, with regards of the tooth greatest length, does not differ from that of other measured fossil specimens.

M1 is densely crowded to P4. By the length and width, M1 resembles the Pleistocene C. lupus from Northern Eurasia (Tab. 4). The paracone is higher than metacone. The protocone is shifted to the anterior margin of talon. The hypocone is diminished. Paraconule is not developed. Metaconule is ridge-like. The lingual cingulum is well differentiated.

M2 is more pronouncedly diminished, with regards to the relative size of this tooth, than in other examined wolves. The ratio between the length of M2 and that of P4 is calculated as 29.9 and $32.1 \%$ respectively $(n=2)$, whereas that of the measured fossil specimens from other regions constitutes $31.2-38.3 \%$ (mean $35.4 \%$, $n=9$ ). This index calculated for the recent wolf from Primorskii Territory varies from $31.2 \%$ to $38.3 \%$ (mean $34.7 \%, n=20)$. M2 is not densely crowded to M1 and more markedly shifted lingual as compared to C. lupus from the Late Pleistocene of Europe (Fig. 3).

Lower teeth. Dimensions of lower incisors: il $(\mathrm{L}=5.9$ $\mathrm{mm}, \mathrm{W}=4.6 \mathrm{~mm}), \mathrm{i} 2(\mathrm{~L}=7.1,7.5,7.9 \mathrm{~mm}, \mathrm{~W}=6.1,6.2$, $6.5 \mathrm{~mm}$, correspondingly; $n=3)$ and $\mathrm{i} 3(\mathrm{~L}=7.3,7.5,7.8$ $\mathrm{mm}$, and $\mathrm{W}=6.6,7.0,8.0 \mathrm{~mm}$, correspondingly; $n=3)$.

Males of the recent wolf from Primorskii Territory have the length of lower canine $12.5-14.5 \mathrm{~mm}$ and width $8.0-9.6 \mathrm{~mm}(n=10)$. The female canine length is $11.1-12.8 \mathrm{~mm}$ and width $7.5-8.3 \mathrm{~mm}(n=7)$. The fossil sample is found to involve 9 male lower canines with the length exceeding $13.0 \mathrm{~mm}(13.0-15.2 \mathrm{~mm})$ and width exceeding $8.5 \mathrm{~mm}(8.5-9.8 \mathrm{~mm})$. Females were recognized by four canines $(\mathrm{L}=11.8-13.0 \mathrm{~mm}, \mathrm{~W}=8.1-$ $8.2 \mathrm{~mm}$ ).

The lower premolar $\mathrm{p} 1$ is single-cuspid ( $\mathrm{L}=5.8-6.8$ $\mathrm{mm}, \mathrm{W}=5.5-5.3 \mathrm{~mm}, n=3)$. The premolar $\mathrm{p} 2$ reveals 
Table 3. Measurements ( $\mathrm{mm}$ ) of mandibles of Late Pleistocene Canis lupus.

\begin{tabular}{|c|c|c|c|c|c|c|c|c|c|c|c|}
\hline \multirow[t]{2}{*}{$\begin{array}{l}\text { Measure- } \\
\text { ments }\end{array}$} & \multicolumn{6}{|c|}{ Geographical Society Cave, Russia } & \multirow{2}{*}{$\begin{array}{c}\text { Obman- } \\
\text { chivoye, } \\
\text { Yakutia, } \\
\text { Russia } \\
\text { GMY } \\
5171\end{array}$} & \multicolumn{2}{|c|}{$\begin{array}{l}\text { Gailenreuth, } \\
\text { Germany }\end{array}$} & \multicolumn{2}{|c|}{$\begin{array}{c}\text { Srbsko Chlum- } \\
\text { Komín Cave, Czech } \\
\text { Republic }\end{array}$} \\
\hline & $\begin{array}{c}\text { ZIN } \\
37268 \\
\end{array}$ & $\begin{array}{c}\text { ZIN } \\
37269 \\
\end{array}$ & $\begin{array}{c}\text { ZIN } \\
37273 \\
\end{array}$ & $\begin{array}{c}\text { ZIN } \\
37270 \\
\end{array}$ & $\begin{array}{c}\mathrm{ZIN} \\
37272 \\
\end{array}$ & $\begin{array}{c}\mathrm{ZIN} \\
37271 \\
\end{array}$ & & $\begin{array}{l}\text { NHM } \\
404 \mathrm{c}\end{array}$ & $\begin{array}{c}\text { NHM } \\
403 a \\
\end{array}$ & $\begin{array}{l}\text { NHMP } \\
\text { R3720 }\end{array}$ & $\begin{array}{l}\text { NHMP } \\
\text { R5159 }\end{array}$ \\
\hline $\mathrm{Lc} 1-\mathrm{m} 3$ & 123.8 & & & & & & & 117.8 & & 116.6 & 113.7 \\
\hline Lp1-m3 & 99.2 & & 101.8 & & & & & 97.5 & 103.0 & & \\
\hline Lp2-m2 & 87.8 & 87.8 & 89.7 & 88.4 & 86.0 & 87.6 & & 85.4 & 85.1 & & \\
\hline Lp1--p4 & 52.8 & 34.6 & 55.5 & 54.9 & & & & 52.3 & 53.7 & & \\
\hline $\mathrm{Lm} 1-\mathrm{m} 3$ & 46.5 & & 48.3 & & 47.3 & & & 47.7 & & 45.6 & 44.8 \\
\hline $\begin{array}{c}\text { Height } \\
\text { behind } \mathrm{m} 1\end{array}$ & 36.8 & 35.0 & 35.9 & & 32.4 & & 34.7 & 34.0 & 31.0 & & \\
\hline $\begin{array}{c}\text { Height } \\
\text { behind p2 }\end{array}$ & 30.1 & 27.4 & 29.5 & 30.1 & 28.1 & 28.7 & 29.7 & 26.0 & 27.2 & 26.3 & 28.5 \\
\hline Teeth & & & & & & & & & & & \\
\hline Lc1 & 14.5 & & & 15.7 & & & 14.6 & & & & 12.7 \\
\hline $\mathrm{Wc1}$ & 10.3 & & & 10.1 & & & 9.1 & & & & 8.2 \\
\hline Lp1 & 6.0 & & & & & & & & 5.5 & & \\
\hline Wp1 & 5.1 & & & & & & & & 4.9 & & \\
\hline Lp2 & 12.7 & 13.1 & & 13.8 & 13.1 & 14.1 & 12.4 & 13.1 & 12.8 & 13.7 & 12.8 \\
\hline Wp2 & 6.7 & 6.4 & & 6.6 & 6.1 & 6.5 & 7.1 & 7.0 & 6.3 & 6.6 & 6.1 \\
\hline Lp3 & 14.2 & 15.3 & & 15.3 & & 15.0 & & 14.1 & 14.4 & 14.8 & 14.2 \\
\hline Wp3 & 7.1 & 7.4 & & 7.6 & & 7.4 & & 7.4 & 7.1 & 7.2 & 6.5 \\
\hline Lp4 & 16.1 & 17.1 & & 16.9 & & 16.9 & 15.3 & 17.1 & 15.8 & 16.9 & 16.0 \\
\hline Wp4 & 8.9 & 8.4 & & 8.1 & & 8.4 & 8.6 & 8.9 & 8.1 & 8.7 & 7.9 \\
\hline $\mathrm{Lm} 1$ & 30.7 & - & 30.3 & 31.3 & 28.6 & 30.5 & 29.8 & 30.4 & 28.1 & 28.9 & 28.1 \\
\hline Ltldm 1 & 7.1 & 6.4 & 8.7 & 7.7 & 8.1 & 8.3 & 6.3 & 8.6 & 7.3 & 6.1 & 6.0 \\
\hline $\mathrm{Wm} 1$ & 11.8 & 11.9 & 12.5 & 12.2 & 12.3 & 12.3 & 12.3 & 12.6 & 12.1 & 12.0 & 11.0 \\
\hline $\mathrm{Lm} 2$ & 11.5 & 12.1 & & 11.6 & 11.9 & 11.6 & 11.5 & 11.4 & 11.2 & 10.0 & 11.6 \\
\hline $\mathrm{Wm} 2$ & 9.0 & 9.2 & & 8.3 & 9.1 & 8.7 & 8.6 & 8.8 & 8.1 & 8.5 & 7.9 \\
\hline
\end{tabular}

Table 4. Measurements (mm) of upper cheek teeth of Late Pleistocene Canis lupus.

\begin{tabular}{|c|c|c|c|c|c|c|c|c|c|c|}
\hline Localities & $\begin{array}{l}\text { Museum } \\
\text { number }\end{array}$ & LP3 & WP3 & LP4 & LpaP4 & WP4 & LM1 & WM1 & LM2 & WM2 \\
\hline \multirow{5}{*}{$\begin{array}{l}\text { Geographical } \\
\text { Society Cave, } \\
\text { Russia }\end{array}$} & ZIN 37267 & & & 25.5 & 17.3 & 15.0 & 16.2 & 19.8 & 8.2 & 11.0 \\
\hline & ZIN 37266 & & & 25.4 & 16.7 & 13.4 & 15.7 & 18.2 & 7.6 & 10.6 \\
\hline & ZIN 37274-3 & 18.9 & 8.4 & 28.0 & 19.3 & 16.0 & & & & \\
\hline & ZIN 37274-2 & 15.7 & 7.3 & & & & & & & \\
\hline & ZIN 37274-4 & & & & & & & & 9.6 & 12.4 \\
\hline \multirow{2}{*}{$\begin{array}{l}\text { Khaptashinsky } \\
\text { Yar, Siberia, } \\
\text { Russia }\end{array}$} & GMY 3728 & 17.5 & 7.3 & 26.7 & - & 13.4 & 16.2 & 20.5 & 9.5 & 12.9 \\
\hline & GMY 3729 & & & & & & 16.5 & 19.4 & 8.2 & 11.8 \\
\hline $\begin{array}{l}\text { Omolon River, } \\
\text { Siberia, Russia }\end{array}$ & SESC 10 & 17.0 & 7.5 & 25.1 & - & 15.5 & 16.3 & 20.4 & 9.2 & 11.9 \\
\hline $\begin{array}{l}\text { Karmalki, } \\
\text { Russia }\end{array}$ & GMMK n/n & 16.3 & 7.6 & 24.8 & 17.2 & 13.2 & 17.0 & 20.3 & 9.5 & 14.7 \\
\hline $\begin{array}{l}\text { Kostenki, } \\
\text { Russia }\end{array}$ & ZIN 36233 & & & 27.2 & 18.4 & 14.6 & 17.6 & 20.6 & & \\
\hline \multirow{6}{*}{$\begin{array}{l}\text { Zoolithen } \\
\text { (Gailenreuth), } \\
\text { Germany }\end{array}$} & NHM 404b & & & 26.8 & - & 16.3 & 17.8 & 21.0 & 9.7 & 12.6 \\
\hline & NHM 403 & & & 23.8 & - & 15.0 & 15.9 & 18.0 & 7.9 & 10.7 \\
\hline & NHM 24 & & & 24.9 & - & 12.4 & 15.8 & 20.0 & & \\
\hline & NHMB 2001 & 17.4 & 8.3 & & & & 16.6 & 20.3 & 10.0 & 12.0 \\
\hline & NHMB 28929 & 16.3 & 7.2 & 26.4 & 17.9 & 14.5 & 17.2 & 20.0 & 8.9 & 12.4 \\
\hline & NHMB 30370 & 15.4 & 6.5 & 23.9 & 16.5 & 13.1 & 15.8 & 19.5 & 8.3 & 12.0 \\
\hline $\begin{array}{l}\text { Wierzchowska } \\
\text { Górna, Poland }\end{array}$ & ISAK 6029 & 17.4 & 7.0 & 25.1 & 16.5 & 15.7 & 16.5 & 21.1 & 9.2 & 13.7 \\
\hline \multirow{5}{*}{$\begin{array}{l}\text { Srbsko Chlum- } \\
\text { Komín Cave, } \\
\text { Czech Republic }\end{array}$} & NHMP R3717 & 17.7 & 7.2 & 26.6 & 17.8 & 15.2 & 16.4 & 19.7 & 8.3 & 11.8 \\
\hline & NHMP 5229 & & & 25.2 & 17.0 & 15.6 & & & 9.2 & 12.6 \\
\hline & NHMP 5228 & & & 25.8 & 17.2 & 15.5 & 16.7 & 19.7 & 8.8 & 11.5 \\
\hline & NHMP 504 & & & & & & 18.0 & 21.3 & 8.9 & 13.8 \\
\hline & NHMP 4161 & 16.9 & 6.6 & & & & & & & \\
\hline
\end{tabular}



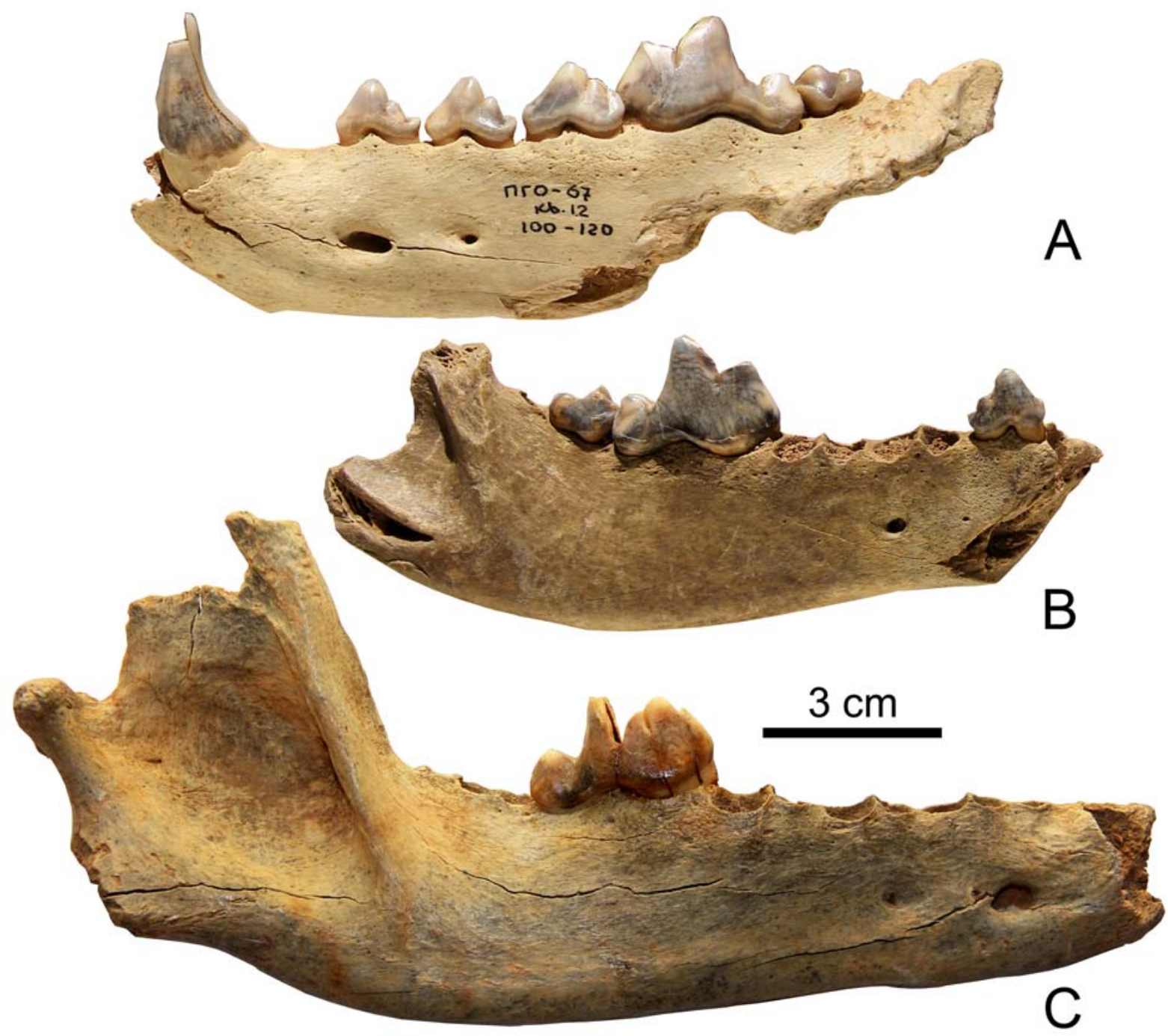

Fig. 4. Canis lupus, left (A) and right (B, C) mandibles from Geographical Society Cave, buccal views. A - ZIN 37270; B - ZIN 37272; C - ZIN 37373.

the posterior accessory cusp (well-defined in 4 specimens and poorly developed in 6 specimens). All specimens of $\mathrm{p} 3$ show posterior accessory cusp and distinct postcingulid. Premolar 4 is larger in comparison with p2 and p3 and reveals higher located posterior accessory cusp. The principal cusp is lower than paraconid of $\mathrm{m} 1$ (Fig. 4A). Small additional tubercle is visible. Dimensions of premolars are given in Tab. 4.

The length of lower carnassial $\mathrm{m} 1$ in the collection from Geographical Society Cave varies from $28.3 \mathrm{~mm}$ to $30.8 \mathrm{~mm}(n=11)$ (Tab. 5), which corresponds to this dimension range in the recent wolf from Primorskii Territory (26.2-30.4 mm, $\mathrm{n}=18)$. However, the mean value of this length in the fossil sample $(29.50 \mathrm{~mm})$ surpasses that of the recent animals $(27.72 \mathrm{~mm})$. The explanation of this seems to be a noticeable difference in male/female ratio in compared samples - the fossil sample consists mainly from the male teeth. Males of the recent wolf from Primorskii Territory have the lower carnassial measured $26.2-30.4 \mathrm{~mm}$ in length $(n=9)$, whereas females exhibit the length of this tooth 26.2-27.9 $\mathrm{mm}(n=9)$. The fossil sample provisionally includes 8 male specimens, with the length exceeding $28.8 \mathrm{~mm}$, and 3 female teeth.

The fossil $\mathrm{m} 1$ is shaped typically of C. lupus (Fig. 5). Paraconid and protoconid are apically pointed. The metaconid is well developed, locating near the posterior margin of protoconid. The talonid constitutes less than $1 / 3$ of the tooth greatest length (22.7-30.9\%, mean $26.0 \%, n=10)$. The talonid of the recent-wolf specimens from Primorskii Territory was found to be somewhat longer $(23.7-34.3 \%$, mean $28.8 \%, n=18)$. The talonid basin is deep and opened lingual. The talonid cusps, hypoconid and entoconid, are distinct, singlepointed; the hypoconid is markedly higher than entoconid. The hypoconid is directly adjoined to the poste- 
Table 5. Measurements ( $\mathrm{mm}$ ) of lower cheek teeth of Late Pleistocene Canis lupus.

\begin{tabular}{|c|c|c|c|c|c|c|c|c|c|c|c|c|}
\hline Localities & $\begin{array}{c}\text { Museum } \\
\text { number }\end{array}$ & $\mathrm{Lp} 2$ & $\mathrm{Wp} 2$ & Lp3 & Wp3 & Lp4 & Wp4 & Lm1 & Ltldm1 & Wm1 & $\operatorname{Lm} 2$ & $\mathrm{Wm} 2$ \\
\hline \multirow{22}{*}{$\begin{array}{l}\text { Geogra- } \\
\text { phical } \\
\text { Society } \\
\text { Cave, } \\
\text { Russia }\end{array}$} & ZIN 37268 & 13.9 & 6.5 & 15.0 & 7.3 & 17.1 & 8.2 & 30.8 & 7.0 & 12.5 & 12.0 & 9.3 \\
\hline & ZIN 37269 & 13.4 & 6.4 & 15.5 & 7.6 & 17.1 & 8.5 & - & 6.2 & 12.2 & 11.8 & 9.2 \\
\hline & ZIN 37273 & & & & & & & 30.4 & 8.3 & 12.6 & & \\
\hline & ZIN 37270 & 14.0 & 6.6 & 15.2 & 7.3 & 17.0 & 8.0 & 30.7 & 7.6 & 12.2 & 11.4 & 8.4 \\
\hline & ZIN 37272 & 13.1 & 6.6 & & & & & 28.5 & 8.3 & 12.2 & 12.3 & 8.8 \\
\hline & ZIN 37271 & 14.1 & 6.5 & 14.9 & 7.4 & 17.0 & 8.5 & 30.9 & 7.8 & 12.3 & 11.6 & 8.7 \\
\hline & ZIN 37274-5 & 14.3 & 7.1 & 16.3 & 8.1 & & & & & & & \\
\hline & ZIN 37274-7 & 13.1 & 6.4 & 15.5 & 7.6 & 17.5 & 9.3 & & & & & \\
\hline & ZIN 37274-8 & 13.4 & 6.6 & 14.8 & 7.2 & 17.2 & 8.4 & & & & & \\
\hline & ZIN 37274-9 & 13.2 & 6.5 & & & 16.7 & 8.5 & & & & & \\
\hline & ZIN 37274-6 & 13.7 & 7.3 & & & & & & & & & \\
\hline & ZIN 37274-254 & & & 14.3 & 7.2 & & & & & & & \\
\hline & ZIN 37274-257 & & & 15.0 & 8.1 & & & & & & & \\
\hline & ZIN 37274-17 & & & 14.7 & 7.6 & 17.0 & 8.6 & & & & & \\
\hline & ZIN 37274-10 & & & & & 16.4 & 8.4 & 28.8 & 7.3 & 12.4 & 11.0 & 8.0 \\
\hline & ZIN 37274-11 & & & & & 15.9 & 7.8 & 29.0 & 6.7 & 11.1 & & \\
\hline & ZIN 37274-12 & & & & & 17.0 & 8.6 & 29.4 & - & 12.9 & & \\
\hline & ZIN 37274-19 & & & & & 16.0 & 9.5 & & & & & \\
\hline & ZIN 37274-13 & & & & & & & 28.3 & 8.2 & 11.3 & & \\
\hline & ZIN 37274-15 & & & & & & & - & 7.4 & 13.1 & 11.9 & 8.5 \\
\hline & ZIN 37274-16 & & & & & & & 29.3 & 6.9 & 11.9 & 12.3 & 8.9 \\
\hline & ZIN 37274-22 & & & & & & & 28.4 & 8.0 & 11.0 & & \\
\hline \multirow{9}{*}{$\begin{array}{l}\text { Zoolithen } \\
\text { (Gailen- } \\
\text { reuth), } \\
\text { Germany }\end{array}$} & NHM 403a & 12.8 & 6.3 & 14.4 & 7.1 & 15.8 & 8.1 & 28.1 & 7.3 & 12.1 & 11.2 & 8.1 \\
\hline & NHM 404 & 12.6 & 6.3 & 14.3 & 7.1 & 16.5 & 8.5 & 29.0 & 7.2 & 12.1 & 11.6 & 8.9 \\
\hline & NHM 404c & 13.1 & 7.0 & 14.1 & 7.4 & 17.1 & 8.9 & 30.4 & 8.6 & 12.6 & 11.5 & 8.6 \\
\hline & NHM 23 & & & 13.7 & 5.9 & & & 27.8 & 7.1 & 11.1 & 11.8 & 8.0 \\
\hline & NHM 403b & & & & & & & 29.7 & 8.0 & 11.4 & & \\
\hline & NHMB 2001 & & & 14.9 & 7.3 & 16.1 & 8.9 & 30.0 & 7.3 & 11.6 & 11.5 & 8.6 \\
\hline & NHMB 28962 & & & 15.1 & 7.6 & 17.7 & 9.1 & 30.5 & 8.1 & 12.9 & 12.3 & 9.5 \\
\hline & NHMB 28963 & 12.4 & 5.9 & 14.8 & 7.0 & 16.8 & 8.4 & 30.6 & 9.0 & 11.6 & & \\
\hline & NHMB 30370 & & & & & 17.0 & 8.7 & ca28.0 & 7.4 & 11.7 & 11.6 & 8.7 \\
\hline \multirow{5}{*}{$\begin{array}{l}\text { Srbsko } \\
\text { Chlum- } \\
\text { Komín } \\
\text { Cave, } \\
\text { Czech } \\
\text { Republic }\end{array}$} & NHMP 3720 & 13.7 & 6.6 & 14.8 & 7.2 & 16.9 & 8.7 & 28.9 & 6.1 & 12.0 & 12.0 & 8.5 \\
\hline & NHMP 4254 & 13.1 & 6.1 & 14.6 & 6.5 & & & 27.7 & 7.2 & 10.9 & & \\
\hline & NHMP 6265 & 13.1 & 6.4 & 14.6 & 6.7 & 16.6 & 8.4 & 28.4 & 7.5 & 12.0 & 11.2 & 8.5 \\
\hline & NHMP 5159 & 12.8 & 6.1 & 14.2 & 6.5 & 16.0 & 7.9 & 28.1 & 6.0 & 11.0 & 11.6 & 7.9 \\
\hline & NHMP 5158 & 13.0 & 5.9 & 14.3 & 6.4 & 16.0 & 7.7 & & & & 11.6 & 7.3 \\
\hline
\end{tabular}

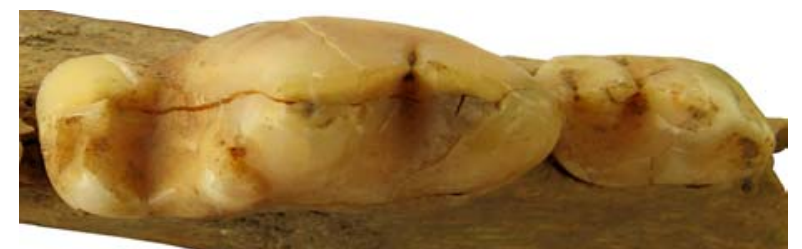

$$
1 \mathrm{~cm}
$$

A

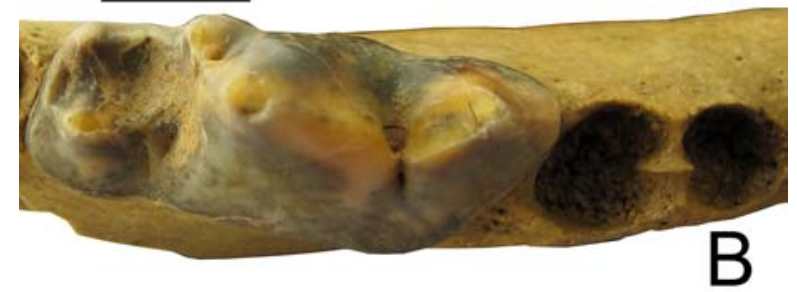

Fig. 5. Canis lupus, lower teeth $\mathrm{p} 4$ and $\mathrm{m} 1$ from Geographical Society Cave, occlusal view.

A - ZIN 37274-11, left; B - ZIN 37274-13, right. rior margin of protoconid $(n=12)$ or shifted backwards with respect to the latter $(n=5)$. Entoconulid is absent. Two specimens having, nevertheless, a miniature accessory cuspid in front of the entoconid are present. The place of hypoconulid is detected by a transverse ridge.

The crown of $\mathrm{m} 2$ is characterized in the fossil material by the robust protoconid. The metaconid is lower and shifted backwards. The hypoconid, which is present on the talonid, is distanced from the protoconid by a deep valley. Entoconid is not developed. The lingual cingulid is visible in the anterior part of the tooth crown.

The last lower molar $\mathrm{m} 3$ is lost in all mandibles, but its alveoli are detected (excepting a single specimen).

Postcranial bones. Almost all elements of skeleton were found, including cervical vertebrae represented by two atlases and six axes (Tab. 6, Fig. 6). Long bones of limbs are mostly broken; however, several of them as 
Table 6. Measurements ( $\mathrm{mm})$ of cervical vertebrae of Late Pleistocene Canis lupus from Geographical Society Cave, Russia.

\begin{tabular}{|c|c|c|c|c|c|c|c|c|c|}
\hline Museum number & GL & $\mathrm{BFcr}$ & BFed & LAd & $\mathrm{H}$ & LCDe & LAPa & BPacd & SBV \\
\hline \multicolumn{10}{|l|}{ Atlas } \\
\hline ZIN 37274-25 & 49.0 & 48.9 & 37.6 & 19.9 & 33.7 & & & & \\
\hline ZIN 37274-179 & & 44.7 & 37.5 & 20.8 & 33.7 & & & & \\
\hline \multicolumn{10}{|l|}{ Axis } \\
\hline ZIN 37274-26 & & 34.5 & - & & & 60.1 & 60.7 & 36.0 & 24.8 \\
\hline ZIN 37274-180 & & 35.5 & 25.8 & & & 59.5 & 58.9 & ca35 & 23.9 \\
\hline ZIN 37274-181 & & 36.4 & 22.7 & & & 60.7 & 58.2 & - & 27.0 \\
\hline ZIN 37274-182 & & 34.9 & 22.9 & & & 57.2 & 55.9 & 33.1 & 26.7 \\
\hline ZIN 37274-183 & & 40.6 & - & & & - & - & - & 29.2 \\
\hline
\end{tabular}

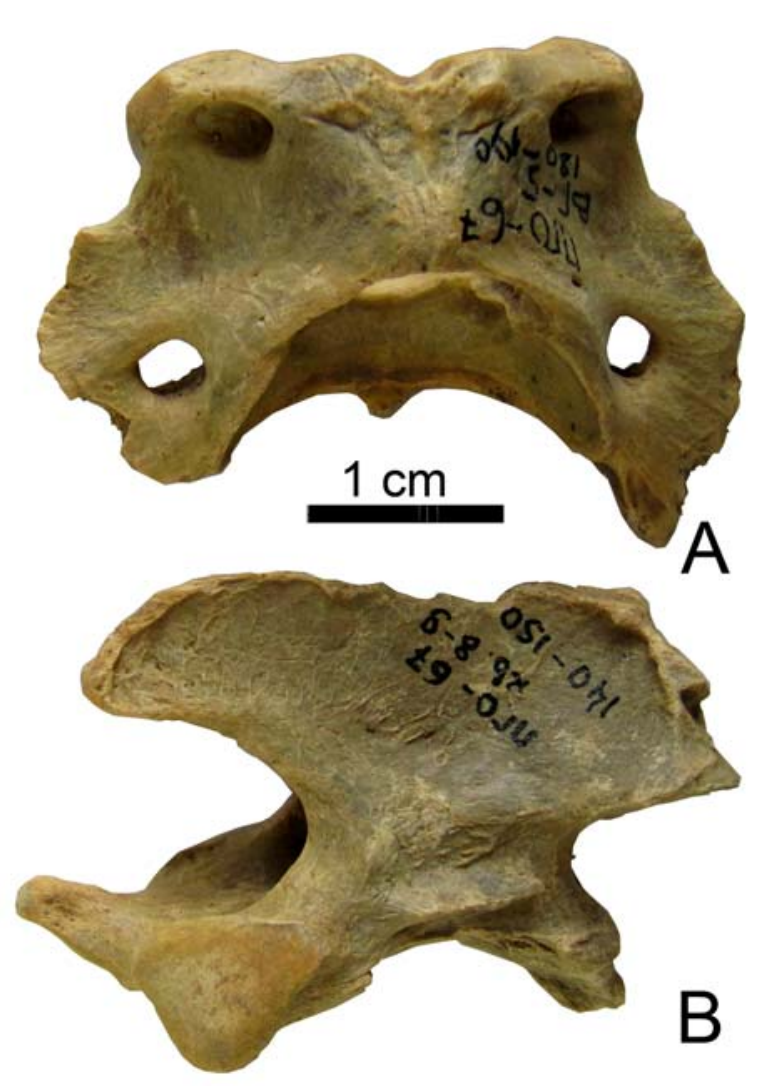

Fig. 6. Canis lupus, cervical vertebrate from Geographical Society Cave, dorsal (A) and lateral (B) views. A - atlas, ZIN 37274-25; B — axis, ZIN 37274-26.

well as many short limb bones remained intact (Fig. 710). These bones do not differ in their shape from corresponding bones of the recent $C$. lupus, being nevertheless more robust (Tab. 7-10).

Comparison. In the tooth size and morphology, the fossil wolf from Geographical Society Cave is similar to the recent subspecies C. lupus coreanus Abe, 1923 from Primorskii Territory, exhibiting however somewhat different proportion of the cranium. In spite of equal length of the lower carnassial with the recent specimens, the fossil mandibles reach a greater height. This suggests the reinforcement of tooth-bone strength

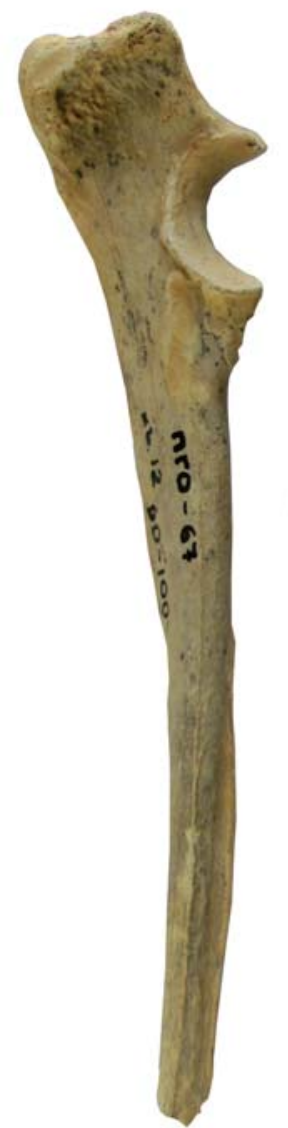

A

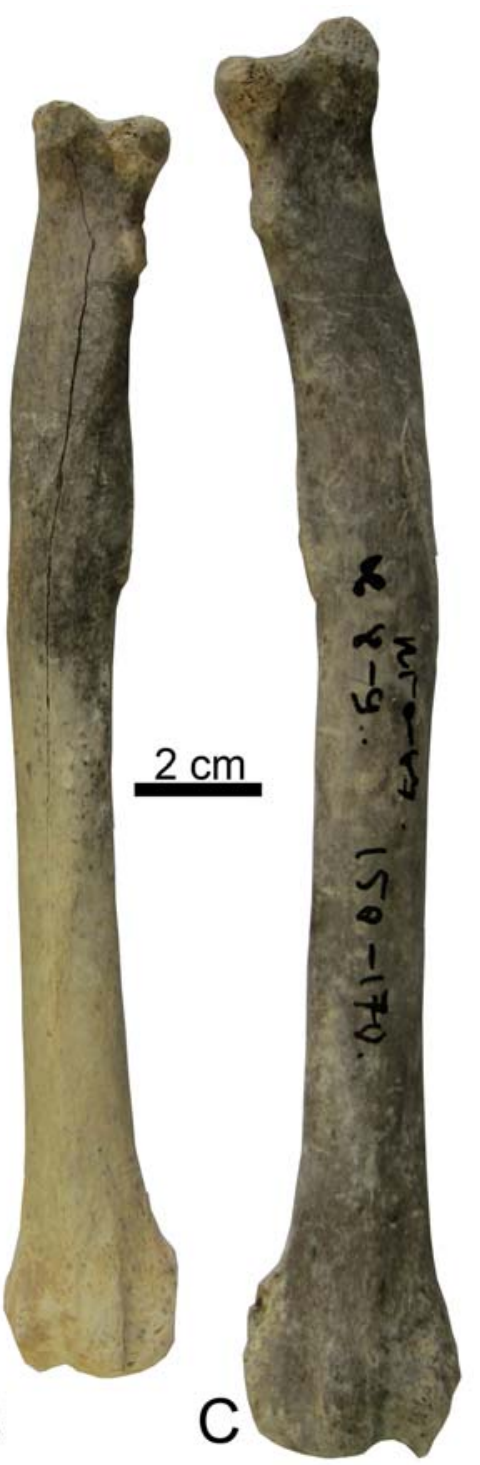

Fig. 7. Canis lupus, hind limb bones from Geographical Society Cave, dorsal $(\mathrm{B}, \mathrm{C})$ and lateral (A) views.

A — ulna, ZIN 37274-27; B — radius, ZIN 37274-29; C — radius, ZIN 37274-28. 

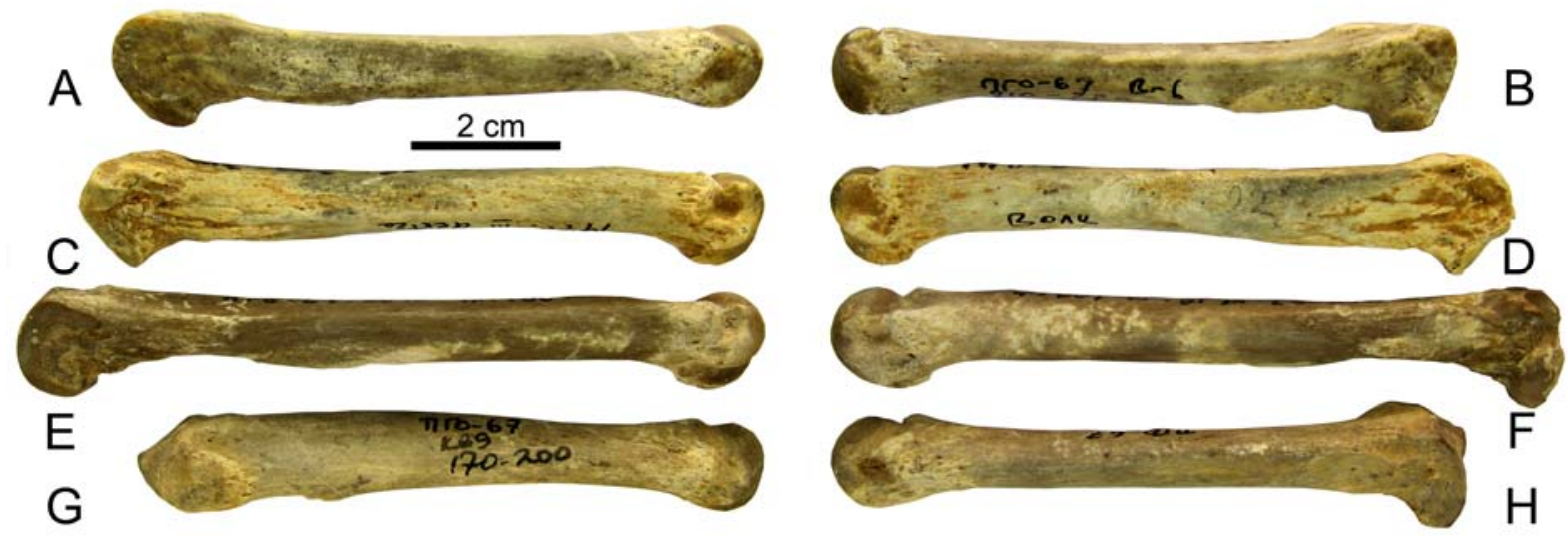

Fig. 8. Canis lupus, right metacarpals from Geographical Society Cave, lateral (A, C, E, G) and medial (B, D, F, H) views. A, B - Mc 2, ZIN 37274-37; C, D - Mc3, ZIN 37274-38; E, F - Mc4, ZIN 37274-39; G, H -Mc5, ZIN 37274-40.

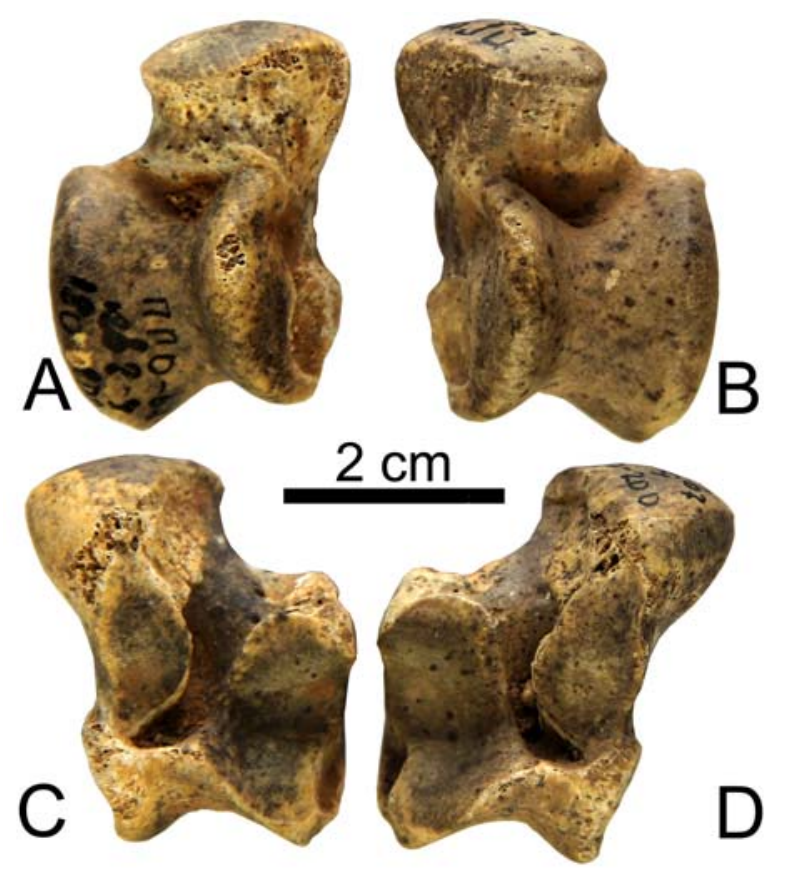

Fig. 9. Canis lupus, talus from Geographical Society Cave, dorsal $(\mathrm{A}, \mathrm{B})$ and ventral $(\mathrm{C}, \mathrm{D})$ views.

A, C - ZIN 37274-43, left; B, D - ZIN 37274-41, right.

and, hence, a capacity to gnawing thicker bones of potential prey. The Late Pleistocene wolf presumably consumed a large carrion in the southern part of the Russian Far East, as it has been already hypothesized for the fossil wolf on the basis of perished mammoths, rhinos, and bison carcasses present here (Leonard et al., 2007; Baryshnikov et al., 2010).

The tooth size of C. lupus, C. lupus variabilis Pei, 1934 and C. chihliensis Zdansky, 1924 from Early and Middle Pleistocene of China (Shanshenmiaozui, Ningyang, Zhoukoudian 1) is considerably smaller than that of the wolf from Geographical Society Cave (Pei, 1934; Zhang, 2001; Tong et al., 2012). Smaller teeth have also C. lupus specimens from the layer $\mathrm{G}$ in Grotta Ramanelli, Italy, which was dated between 69 and 40 thousand years (Sardella et al., 2014).

Taphonomy. Fossil remains of wolf are the most common among the canids in the material from Geographical Society Cave. The minimal number of indi-
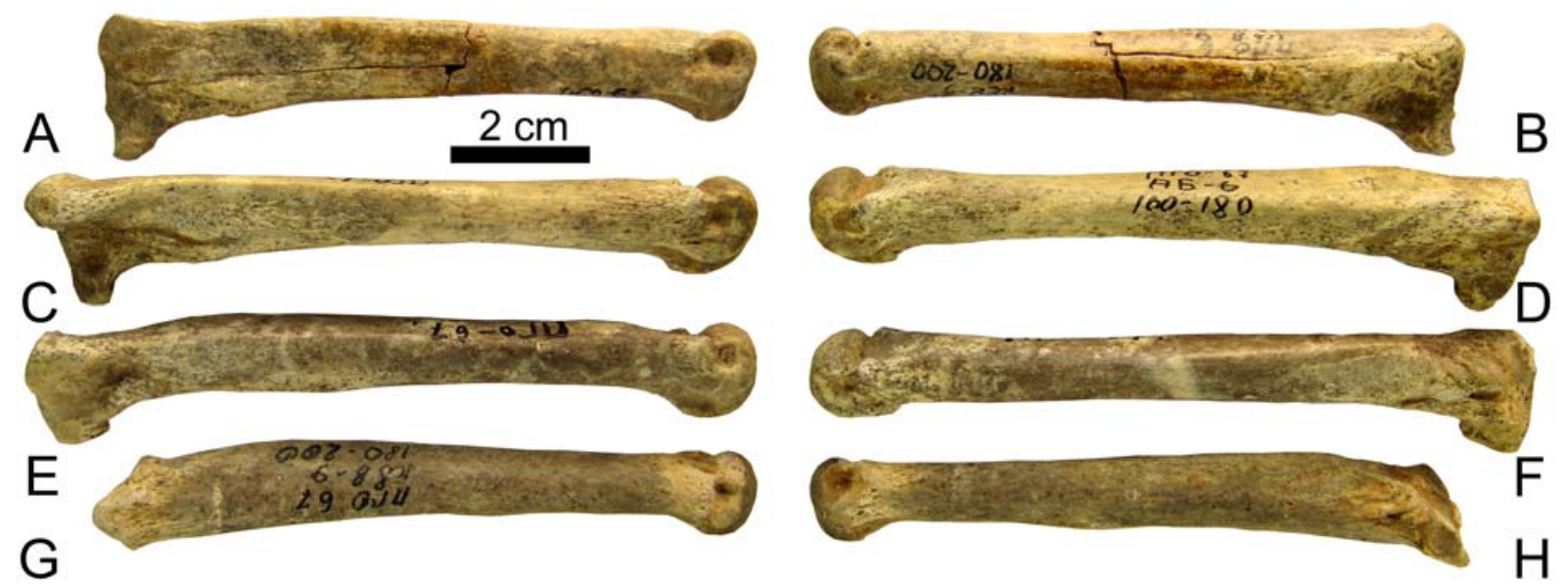

Fig. 10. Canis lupus, right metatarsals from Geographical Society Cave, lateral (A, C, E, G) and medial (B, D, F, H) views. A, B - Mt 2, ZIN 37274-45; C, D — Mt 3, ZIN 37274-46; E, F — Mt4, ZIN 37274-47; G, H - Mt5, ZIN 37274-48. 
Table 7. Measurements (mm) of hind limb bones in Canis lupus.

\begin{tabular}{|c|c|c|c|c|c|c|c|c|c|c|}
\hline Locality & Museum number & GL & $\mathrm{Bp}$ & SD & $\mathrm{Bd}$ & SLC & GLP & BG & SDO & DPA \\
\hline \multicolumn{11}{|c|}{ Scapula } \\
\hline \multirow{5}{*}{$\begin{array}{l}\text { Geographical Society Cave, } \\
\text { Russia }\end{array}$} & ZIN 37274-185 & & & & & 34.6 & 41.5 & 26.3 & & \\
\hline & ZIN 37274-186 & & & & & 34.8 & 40.7 & 25.2 & & \\
\hline & ZIN 37274-187 & & & & & 32.1 & 35.1 & 21.5 & & \\
\hline & ZIN 37274-189 & & & & & 29.1 & 37.4 & 22.0 & & \\
\hline & ZIN 37274-255 & & & & & & 33.6 & 21.6 & & \\
\hline $\begin{array}{l}\text { Srbsko Chlum-Komín } \\
\text { Cave, Czech Republic }\end{array}$ & NHMP R5383 & & & & & 33.4 & 41.9 & 26.4 & & \\
\hline \multicolumn{11}{|c|}{ Humerus } \\
\hline \multirow{6}{*}{$\begin{array}{l}\text { Geographical Society Cave, } \\
\text { Russia }\end{array}$} & ZIN 37274-192 & & & 17.7 & 44.4 & & & & & \\
\hline & ZIN 37274-193 & & & & 41.9 & & & & & \\
\hline & ZIN 37274-196 & & & & 45.8 & & & & & \\
\hline & ZIN 37274-195 & & & & 43.0 & & & & & \\
\hline & ZIN 37274-197 & & & & 40.3 & & & & & \\
\hline & ZIN 37274-198 & & & & 39.2 & & & & & \\
\hline \multirow{2}{*}{$\begin{array}{l}\text { Srbsko Chlum-Komín } \\
\text { Cave, Czech Republic }\end{array}$} & NHMP R5162 & 200.4 & & 15.9 & 40.3 & & & & & \\
\hline & NHMP R5183 & 201.2 & & 16.0 & 40.3 & & & & & \\
\hline \multicolumn{11}{|c|}{ Ulna } \\
\hline $\begin{array}{l}\text { Geographical Society Cave, } \\
\text { Russia }\end{array}$ & ZIN 37274-27 & & & & & & & & 27.8 & 32.3 \\
\hline $\begin{array}{l}\text { Srbsko Chlum-Komín } \\
\text { Cave, Czech Republic }\end{array}$ & NHMP R5387 & & & & & & & & 27.6 & 34.8 \\
\hline \multicolumn{11}{|c|}{ Radius } \\
\hline \multirow{4}{*}{$\begin{array}{l}\text { Geographical Society Cave, } \\
\text { Russia }\end{array}$} & ZIN 37274-29 & 195.7 & 21.7 & 16.4 & 28.7 & & & & & \\
\hline & ZIN 37274-28 & 218.2 & 25.6 & 18.9 & 35.0 & & & & & \\
\hline & ZIN 37274-206 & & 24.8 & 17.2 & & & & & & \\
\hline & ZIN 37274-258 & & & 16.8 & & & & & & \\
\hline \multirow{2}{*}{$\begin{array}{l}\text { Srbsko Chlum-Komín } \\
\text { Cave, Czech Republic }\end{array}$} & NHMP R5170 & 198.4 & 22.8 & 16.8 & 30.4 & & & & & \\
\hline & NHMP R5386 & 198.5 & 25.3 & 17.5 & 32.8 & & & & & \\
\hline Mongolia, recent, $q$ & ZIN 24365 & 199.6 & 19.1 & 14.1 & 26.9 & & & & & \\
\hline
\end{tabular}

Table 8. Measurements ( $\mathrm{mm}$ ) of metacarpals in Canis lupus (to be continued).

\begin{tabular}{|c|c|c|c|c|c|c|c|}
\hline Locality & Museum number & GL & $\mathrm{Bp}$ & $\mathrm{Dp}$ & SD & $\mathrm{Bd}$ & $\mathrm{Dd}$ \\
\hline \multicolumn{8}{|c|}{ Mc2 } \\
\hline \multirow{11}{*}{ Geographical Society Cave, Russia } & ZIN 37274-37 & 79.7 & 11.9 & 15.2 & 10.1 & 13.2 & 11.0 \\
\hline & ZIN 37274-55 & 84.6 & 12.5 & 16.0 & 10.0 & 13.4 & 12.7 \\
\hline & ZIN 37274-56 & 77.8 & 10.5 & 14.2 & 9.2 & 12.4 & 11.1 \\
\hline & ZIN 37274-57 & 81.5 & 10.9 & 15.5 & 9.4 & 13.9 & 11.3 \\
\hline & ZIN 37274-58 & 85.6 & 13.6 & 16.0 & 11.0 & 14.1 & 13.1 \\
\hline & ZIN 37274-59 & 84.5 & 12.5 & 15.5 & 10.7 & 13.9 & 12.5 \\
\hline & ZIN 37274-60 & & 10.4 & 13.3 & 8.4 & & \\
\hline & ZIN 37274-61 & 78.5 & 10.3 & 13.8 & 8.8 & 12.1 & 10.4 \\
\hline & ZIN 37274-62 & & 9.6 & 13.8 & 9.3 & & \\
\hline & ZIN 37274-229 & 76.9 & 10.7 & 13.4 & 8.6 & 11.9 & 10.4 \\
\hline & ZIN 37274-230 & 81.1 & 11.6 & 14.6 & 9.0 & 12.7 & 11.0 \\
\hline \multirow{5}{*}{ Gailenreuth Cave, Germany } & NHM 222 & 76.7 & & & & 13.3 & \\
\hline & NHM 222 & 72.8 & & & & 12.9 & \\
\hline & NHM 403 & 73.2 & & & & 11.6 & \\
\hline & NHM 403 & 76.8 & & & & 12.8 & \\
\hline & NHM 403 & 80.7 & & & & 13.3 & \\
\hline $\begin{array}{l}\text { Srbsko Chlum-Komín Cave, Czech } \\
\text { Republic }\end{array}$ & NHMP R5214 & 75.7 & 11.0 & 13.4 & 8.2 & 12.0 & 10.9 \\
\hline Mongolia, recent, $q$ & ZIN 24365 & 73.7 & 10.1 & 12.2 & 7.0 & 10.6 & 9.5 \\
\hline
\end{tabular}


Table 8 (continued).

\begin{tabular}{|c|c|c|c|c|c|c|c|}
\hline Locality & Museum number & GL & $\mathrm{Bp}$ & $\mathrm{Dp}$ & SD & $\mathrm{Bd}$ & Dd \\
\hline \multicolumn{8}{|c|}{ Mc3 } \\
\hline \multirow{9}{*}{ Geographical Society Cave, Russia } & ZIN 37274-38 & 86.7 & 12.1 & 15.6 & 9.4 & 12.6 & 12.8 \\
\hline & ZIN 37274-76 & & 11.3 & 14.6 & 9.3 & & \\
\hline & ZIN 37274-77 & & 11.2 & 14.7 & 8.7 & & \\
\hline & ZIN 37274-37 & 87.6 & 11.9 & 15.7 & 9.2 & 12.6 & 12.9 \\
\hline & ZIN 37274-78 & 90.0 & 11.5 & 14.8 & 8.6 & 11.7 & 12.1 \\
\hline & ZIN 37274-79 & 87.7 & 11.1 & 14.6 & 8.6 & 12.1 & 11.7 \\
\hline & ZIN 37274-80 & & 11.9 & 14.5 & 9.7 & & \\
\hline & ZIN 37274-81 & & 11.6 & 15.1 & & & \\
\hline & ZIN 37274-82 & & 10.7 & 14.6 & 8.0 & & \\
\hline \multirow{5}{*}{ Gailenreuth Cave, Germany } & NHM 222 & 86.6 & & & & 11.3 & \\
\hline & NHM 222 & 90.0 & & & & 12.5 & \\
\hline & NHM 222 & 78.1 & & & & 10.5 & \\
\hline & NHM 403 & 98.7 & & & & 13.7 & \\
\hline & NHM 403 & 84.3 & & & & 12.1 & \\
\hline $\begin{array}{l}\text { Srbsko Chlum-Komín Cave, Czech } \\
\text { Republic }\end{array}$ & NHMP R5390 & 88.4 & 13.1 & 16.7 & 8.5 & 12.4 & 13.6 \\
\hline Mongolia, recent, $q$ & ZIN 24365 & 84.2 & 9.8 & 14.0 & 7.1 & 9.7 & 10.5 \\
\hline \multicolumn{8}{|c|}{ Mc4 } \\
\hline \multirow{15}{*}{ Geographical Society Cave, Russia } & ZIN 37274-64 & 94.2 & 11.8 & 17.0 & 9.7 & 12.6 & 14.0 \\
\hline & ZIN 37274-65 & 90.5 & 11.3 & 14.4 & 9.2 & 12.0 & 12.7 \\
\hline & ZIN 37274-66 & 88.9 & 10.3 & 15.3 & 8.3 & 11.2 & 12.0 \\
\hline & ZIN 37274-63 & 98.1 & 11.4 & 14.3 & 7.8 & 10.4 & 11.4 \\
\hline & ZIN 37274-67 & & 10.0 & 17.3 & 8.5 & & \\
\hline & ZIN 37274-68 & & 10.8 & 17.1 & 9.1 & & \\
\hline & ZIN 37274-73 & & 11.5 & 16.7 & 10.1 & & \\
\hline & ZIN 37274-69 & 91.9 & 9.7 & 15.4 & 8.3 & 11.6 & 11.7 \\
\hline & ZIN 37274-39 & 95.7 & 12.2 & 16.8 & 9.2 & 12.6 & 13.6 \\
\hline & ZIN 37274-70 & 93.1 & 9.7 & 15.0 & 8.4 & 11.1 & 10.6 \\
\hline & ZIN 37274-71 & 87.0 & 9.6 & 13.8 & 7.7 & 10.8 & 11.7 \\
\hline & ZIN 37274-72 & & 10.0 & 16.6 & 8.7 & & \\
\hline & ZIN 37274-74 & & 9.8 & 14.8 & 8.3 & & \\
\hline & ZIN 37274-83 & 86.2 & 9.4 & 15.1 & 7.9 & 11.8 & \\
\hline & ZIN 37274-75 & & 9.2 & 14.8 & 7.8 & & \\
\hline \multirow{5}{*}{ Gailenreuth Cave, Germany } & NHM 222 & 94.5 & & & & 11.9 & \\
\hline & NHM 222 & 95.1 & & & & 11.4 & \\
\hline & NHM 403 & 81.6 & & & & 11.0 & \\
\hline & NHM 403 & 88.1 & & & & 11.1 & \\
\hline & NHM 403 & 75.7 & & & & 10.9 & \\
\hline \multirow{3}{*}{$\begin{array}{l}\text { Srbsko Chlum-Komín Cave, Czech } \\
\text { Republic }\end{array}$} & NHMP R5213 & 87.3 & 11.5 & 14.8 & 8.0 & 11.4 & 12.3 \\
\hline & NHMP R5214 & 86.9 & 11.2 & 14.5 & 8.3 & 11.5 & 12.2 \\
\hline & NHMP R601 & 89.3 & 11.2 & 14.9 & 8.2 & 12.2 & 12.0 \\
\hline Mongolia, recent, $q$ & ZIN 24365 & 83.8 & 9.0 & 13.7 & 6.4 & 9.3 & 10.6 \\
\hline \multicolumn{8}{|c|}{ Mc5 } \\
\hline \multirow{8}{*}{ Geographical Society Cave, Russia } & ZIN 37274-49 & 72.9 & 13.6 & 14.5 & 9.4 & 13.1 & 11.0 \\
\hline & ZIN 37274-53 & 75.6 & 13.7 & 16.1 & 9.7 & 13.0 & 11.2 \\
\hline & ZIN 37274-54 & & 16.5 & 15.8 & 11.5 & & \\
\hline & ZIN 37274-50 & 76.0 & 13.4 & 13.3 & 9.9 & 12.8 & 10.5 \\
\hline & ZIN 37274-40 & 72.8 & 13.2 & 14.1 & 9.3 & 13.3 & 10.7 \\
\hline & ZIN 37274-51 & & 12.6 & 14.1 & 9.8 & & \\
\hline & ZIN 37274-52 & 69.0 & 14.4 & 12.9 & 9.4 & 12.8 & \\
\hline & ZIN 37274-260 & & & & 9.2 & 10.9 & 10.3 \\
\hline \multirow{4}{*}{ Gailenreuth Cave, Germany } & NHM 222 & 74.9 & & & & 12.9 & \\
\hline & NHM 222 & 72.6 & & & & 12.9 & \\
\hline & NHM 403 & 65.0 & & & & 13.1 & \\
\hline & NHM 403 & 76.0 & & & & 13.5 & \\
\hline $\begin{array}{l}\text { Srbsko Chlum-Komín Cave, Czech } \\
\text { Republic }\end{array}$ & NHMP R5211 & 71.6 & 14.3 & 15.3 & 8.9 & 12.7 & 11.3 \\
\hline Mongolia, recent, $q$ & ZIN 24365 & 72.3 & 12.2 & 11.5 & 8.1 & 10.7 & 10.2 \\
\hline
\end{tabular}


Table 9. Measurements ( $\mathrm{mm}$ ) of hind limb bones in Canis lupus.

\begin{tabular}{|c|c|c|c|c|c|c|c|c|}
\hline Locality & Museum number & GL & $\mathrm{Bp}$ & SD & $\mathrm{Bd}$ & $\mathrm{SH}$ & LAR & GB \\
\hline \multicolumn{9}{|c|}{ Pelvis } \\
\hline \multirow{2}{*}{ Geographical Society Cave, Russia } & ZIN 37274-214 & & & & & 26.8 & 26.5 & \\
\hline & ZIN 37274-213 & & & & & 25.9 & 26.5 & \\
\hline $\begin{array}{l}\text { Srbsko Chlum-Komín Cave, Czech } \\
\text { Republic }\end{array}$ & NHMP R3737 & & & & & 24.6 & 28.5 & \\
\hline \multicolumn{9}{|c|}{ Tibia } \\
\hline \multirow{4}{*}{ Geographical Society Cave, Russia } & ZIN 37274-222 & 217.8 & ca38 & 19.9 & 29.4 & & & \\
\hline & ZIN 37274-223 & & & 18.3 & 32.3 & & & \\
\hline & ZIN 37274-221 & & & 17.4 & 30.6 & & & \\
\hline & ZIN 37274-225 & & & & 30.1 & & & \\
\hline $\begin{array}{l}\text { Srbsko Chlum-Komín Cave, Czech } \\
\text { Republic }\end{array}$ & NHMP R5173 & 219.8 & 45.3 & 17.2 & 29.3 & & & \\
\hline Mongolia, recent, $\stackrel{+}{+}$ & ZIN 24365 & 217.6 & 37.8 & 13.0 & 24.4 & & & \\
\hline \multicolumn{9}{|c|}{ Calcaneus } \\
\hline \multirow{6}{*}{ Geographical Society Cave, Russia } & ZIN 37274-33 & 61.3 & & & & & & 24.4 \\
\hline & ZIN 37274-228 & 62.6 & & & & & & 26.3 \\
\hline & ZIN 37274-32 & 58.5 & & & & & & 26.1 \\
\hline & ZIN 37274-220 & 56.7 & & & & & & 24.3 \\
\hline & ZIN 37274-30 & 53.7 & & & & & & 21.1 \\
\hline & ZIN 37274-32 & 58.3 & & & & & & 26.1 \\
\hline Tigrovaya Cave, Russia & ZIN 65 & 56.7 & & & & & & 22.5 \\
\hline \multirow{2}{*}{$\begin{array}{l}\text { Srbsko Chlum-Komín Cave, Czech } \\
\text { Republic }\end{array}$} & NHMP R4160 & 57.4 & & & & & & 23.0 \\
\hline & NHMP R5314 & 56.2 & & & & & & 22.4 \\
\hline Mongolia, recent,,+ & ZIN 24365 & 50.9 & & & & & & 19.6 \\
\hline \multicolumn{9}{|c|}{ Talus } \\
\hline \multirow{3}{*}{ Geographical Society Cave, Russia } & ZIN 37274-41 & 35.9 & & & & & & 32.4 \\
\hline & ZIN 37274-42 & 34.4 & & & & & & - \\
\hline & ZIN 37274-43 & 34.7 & & & & & & 29.3 \\
\hline Mongolia, recent, $+\rightarrow$ & ZIN 24365 & 30.0 & & & & & & 19.6 \\
\hline
\end{tabular}

viduals is 8 (calculated on the basis of the Mt3). The collection comprises almost all elements of the skeleton, including fragments of vertebrae and ribs; several cervical vertebrae and long bones (two radii and one tibia) as well as many short limb bones are intact (Figs $6-10)$.

Adult individuals predominate (except one subadult represented by a bone fragment with an unfused epiphysis). No juveniles are present. Males are most common (see above). Their prevalence, as well as the absence of juveniles, suggests that Geographical Society Cave was not used by wolves as a den for raising offspring, although these animals often create brood shelters in the Primorskii Territory in rock niches or beneath flat rocks (Yudin, 2013).

Many wolf bones have been gnawed by larger carnivores. Among metapodial bones, gnawed specimens constitute 33\% $(n=88)$. Several mandibles show the lower margin characteristically broken (Fig. 4A). Similar damage was observed on the hyena mandibles (Baryshnikov, 2014). In both cases, mandibles may have been gnawed by hyenas Crocuta ultima (Matsumoto, 1915), which presumably used the cave as a den for protecting their cubs. No traces of gnawing by larger carnivores are observable on the cervical vertebrae.
Eight of the wolf humeri are represented only by the distal end, which occasionally exhibits tooth-marks (Fig. $11 \mathrm{C})$. There is an interesting group of calcanei with traces of gnawing from carnivore teeth on the lateral margin (Fig. 12). The width of the tooth punctures ranges from $4.8 \mathrm{~mm}$ to $8.8 \mathrm{~mm}$ (mean of $6.5 \mathrm{~mm}, n=4$ ), which exceeds dimensions of punctures produced by wolves (Sala et al., 2014). The damage to the humeral and heel bones may have been caused by large carnivores such as hyenas or tigers detaching the distal portions of limbs, which have low value as food. The distal portions of limbs typically remain unbroken in places where tigers have consumed prey (Yudin \& Yudina, 2009). It is therefore not surprising to find large fragments of radii, ulnae, and tibiae, as well as metacarpals, metatarsals and phalanges, intact after being detached from wolf carcasses.

The pattern of preservation is characteristic of natural mortality in a cave or for animals brought to a cave as a prey.

A part of the wolf bones exhibit minute traces of gnawing probably produced by hyena cubs (Fig. 13), which may have gnawed, in their cave-den, bones lying on the cave floor. There is a bone fragment with traces of acidic corrosion, which has been derived from the 
Table 10. Measurements (mm) of metatarsals in Canis lupus.

\begin{tabular}{|c|c|c|c|c|c|c|c|}
\hline Locality & Museum number & GL & $\mathrm{Bp}$ & Dp & SD & $\mathrm{Bd}$ & Dd \\
\hline \multicolumn{8}{|c|}{ Mt2 } \\
\hline \multirow{3}{*}{ Geographic Society Cave, Russia } & ZIN 37274-106 & 80.8 & 11.6 & & 9.3 & 11.3 & 9.0 \\
\hline & ZIN 37274-107 & & 11.5 & 18.7 & 9.2 & & \\
\hline & ZIN 37274-45 & 92.4 & 13.0 & 20.0 & 11.2 & 12.9 & 12.1 \\
\hline \multirow{4}{*}{ Gailenreuth Cave, Germany } & NHM 222 & 89.3 & & & & 12.5 & \\
\hline & NHM 222 & 91.8 & & & & 13.7 & \\
\hline & NHM 403 & 85.0 & & & & 12.0 & \\
\hline & NHM 403 & 87.3 & & & & 13.6 & \\
\hline \multirow{3}{*}{ Srbsko Chlum-Komín Cave, Czech Republic } & NHMP R4200 & 86.6 & 12.2 & 18.1 & 8.7 & 11.5 & 11.0 \\
\hline & NHMP R5199 & 81.0 & 12.3 & 16.5 & 8.5 & 12.9 & 10.7 \\
\hline & NHMP R5187 & 81.0 & 12.3 & 17.1 & 9.6 & 11.7 & 12.0 \\
\hline Mongolia, recent, +9 & ZIN 24365 & 82.0 & 9.7 & 14.9 & 6.1 & 9.9 & 9.4 \\
\hline \multicolumn{8}{|c|}{ Mt3 } \\
\hline \multirow{12}{*}{ Geographic Society Cave, Russia } & ZIN 37274-94 & 104.4 & 14.0 & 18.7 & 9.7 & 13.1 & 12.8 \\
\hline & ZIN 37274-95 & 93.9 & 13.6 & 18.4 & 10.1 & - & 11.2 \\
\hline & ZIN 37274-99 & & 11.6 & 17.3 & 8.8 & & \\
\hline & ZIN 37274-97 & & 13.5 & 18.6 & & & \\
\hline & ZIN 37274-98 & 96.1 & 13.3 & 19.0 & 9.8 & 13.0 & 12.7 \\
\hline & ZIN 37274-96 & 104.1 & 13.3 & 19.5 & 9.7 & 12.3 & 12.0 \\
\hline & ZIN 37274-46 & 103.5 & 14.8 & 19.5 & 10.4 & 13.3 & 14.0 \\
\hline & ZIN 37274-100 & 102.0 & 13.3 & 18.6 & 9.9 & 12.0 & 11.9 \\
\hline & ZIN 37274-101 & 101.2 & & & 10.5 & 13.0 & 13.2 \\
\hline & ZIN 37274-102 & 96.6 & & & 9.9 & 13.3 & \\
\hline & ZIN 37274-103 & & 11.4 & 17.3 & 9.2 & & \\
\hline & ZIN 37274-104 & 91.0 & 11.7 & 16.5 & 9.6 & 12.0 & \\
\hline \multirow{5}{*}{ Gailenreuth Cave, Germany } & NHM 222 & 86.6 & & & & 11.3 & \\
\hline & NHM 222 & 90.0 & & & & 12.5 & \\
\hline & NHM 222 & 78.1 & & & & 10.5 & \\
\hline & NHM 403 & 98.7 & & & & 13.7 & \\
\hline & NHM 403 & 84.3 & & & & 12.1 & \\
\hline \multirow{2}{*}{ Srbsko Chlum-Komín Cave, Czech Republic } & NHMP R5200 & 90.7 & 12.5 & 17.2 & 9.6 & 11.9 & 12.1 \\
\hline & NHMP R5188 & 90.1 & 12.3 & 17.1 & 9.6 & 11.7 & 12.0 \\
\hline Mongolia, recent, +9 & ZIN 24365 & 91.1 & 11.2 & 15.2 & 8.4 & 9.6 & 10.6 \\
\hline \multicolumn{8}{|c|}{ Mt4 } \\
\hline \multirow{4}{*}{ Geographic Society Cave, Russia } & ZIN 37274-93 & & 12.8 & 17.3 & & & \\
\hline & ZIN 37274-47 & 105.3 & 13.6 & 18.6 & 9.9 & 12.6 & 13.4 \\
\hline & ZIN 37274-91 & 106.5 & & & 10.1 & 12.5 & 13.0 \\
\hline & ZIN 37274-92 & & 13.7 & 18.9 & & & \\
\hline \multirow{2}{*}{ Gailenreuth Cave, Germany } & NHM 222 & 101.3 & & & & 11.2 & \\
\hline & NHM 403 & 105.0 & & & & 11.7 & \\
\hline \multirow{5}{*}{ Srbsko Chlum-Komín Cave, Czech Republic } & NHMP R5411 & 85.6 & 9.8 & 13.3 & 7.5 & 11.1 & 12.4 \\
\hline & NHMP R5216 & 96.7 & 11.5 & 15.7 & 7.7 & 11.0 & 12.2 \\
\hline & NHMP R5300 & 81.7 & 10.6 & 17.2 & 9.3 & 12.1 & 13.4 \\
\hline & NHMP R5201 & 92.8 & 11.5 & 15.6 & 9.1 & 11.2 & 11.6 \\
\hline & NHMP R5189 & 93.0 & 11.0 & 16.2 & 8.9 & 10.9 & 11.7 \\
\hline Mongolia, recent, $q$ & ZIN 24365 & 93.2 & 10.6 & 14.5 & 7.1 & 9.3 & 10.3 \\
\hline \multicolumn{8}{|c|}{ Mt5 } \\
\hline \multirow{4}{*}{ Geographic Society Cave, Russia } & ZIN 37274-48 & 97.0 & 12.7 & 15.4 & 9.9 & 12.3 & 12.0 \\
\hline & ZIN 37274-89 & 88.3 & 10.3 & 13.7 & 9.1 & 10.7 & 10.0 \\
\hline & ZIN 37274-90 & 86.8 & 11.4 & 15.5 & 8.6 & 11.9 & 10.9 \\
\hline & ZIN 37277-10 & & 10.8 & 13.4 & 8.9 & & \\
\hline \multirow{4}{*}{ Gailenreuth Cave, Germany } & NHM 222 & 84.9 & & & & 11.6 & \\
\hline & NHM 222 & 86.8 & & & & 11.9 & \\
\hline & NHM 222 & 89.6 & & & & 13.2 & \\
\hline & NHM 403 & 91.6 & & & & 12.2 & \\
\hline \multirow{2}{*}{ Srbsko Chlum-Komín Cave, Czech Republic } & NHMP R5202 & 82.7 & 10.9 & 13.9 & 9.3 & 11.9 & 10.4 \\
\hline & NHMP 5190 & 82.0 & 10.9 & 14.1 & 8.8 & 12.1 & 10.3 \\
\hline Mongolia, recent, +9 & ZIN 24365 & 82.4 & 11.8 & 10.1 & 6.8 & 8.9 & 9.3 \\
\hline
\end{tabular}



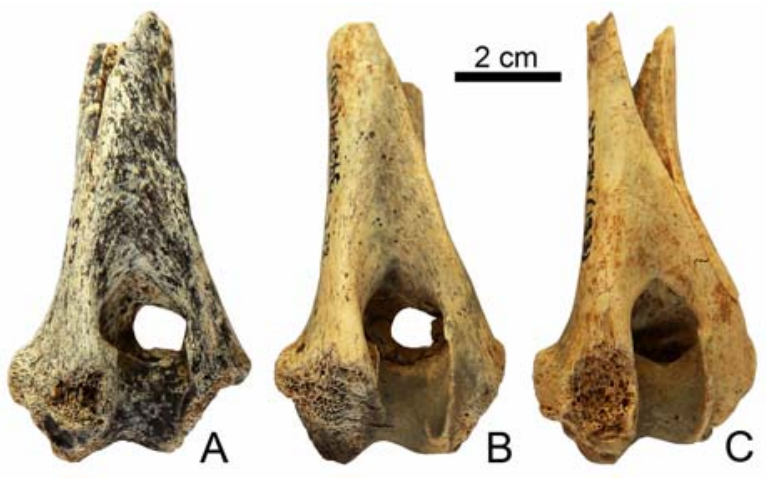

Fig. 11. Canis lupus, distal fragments of humerus, caudal view.

A — ZIN 37274-194, right; B — ZIN 37274-190, right; C — ZIN 37274-193.
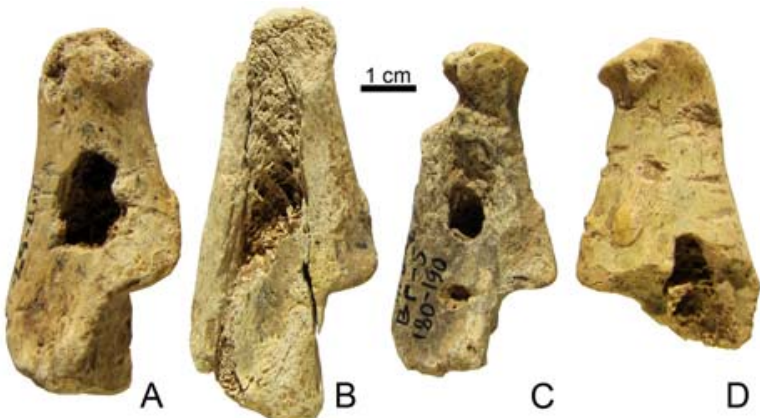

Fig. 12. Canis lupus, calcanei with signs of gnawing by large carnivores (presumably, by hyenas), lateral view. A - ZIN 37274-32, right; B - ZIN 37274-33, right; C - ZIN 37274-31, right; D - ZIN 37274-34, left.

stomach of a hyena. Most probably wolves (alive or dead) were the hyena prey and were brought into the cave by hyenas for consumption or for feeding their cubs. Pleistocene hyenas were larger than wolves, formed clans with complex social behavior, and likely represented dangerous competitors for the canids in the contest for hunting territory and available shelters.

Another potential source of the accumulation of wolf remains in the cave may be the tiger, Panthera tigris (L., 1758) or cave lion, P. spelaea (Goldfuss, 1810), whose bone remains were also found in Geographical Society Cave (Vereshchagin, 1971). Recent wolves are known to abandon tiger habitat in Primorskii Territory, being forced out by a stronger competitor (Kostoglod, 1982). This occurred in Lazovskii Nature Reserve; a tiger killed a wolf but neglected to consume it (Valova et al., 1989). In Pleistocene, wolves, most probably, became the prey of tigers only occasionally; therefore the role of this large cat in the accumulation of wolf bones in the cave was insignificant.

I have not observed reliable signs of modification by stone tools on the wolf bones or examples of burnt specimens.

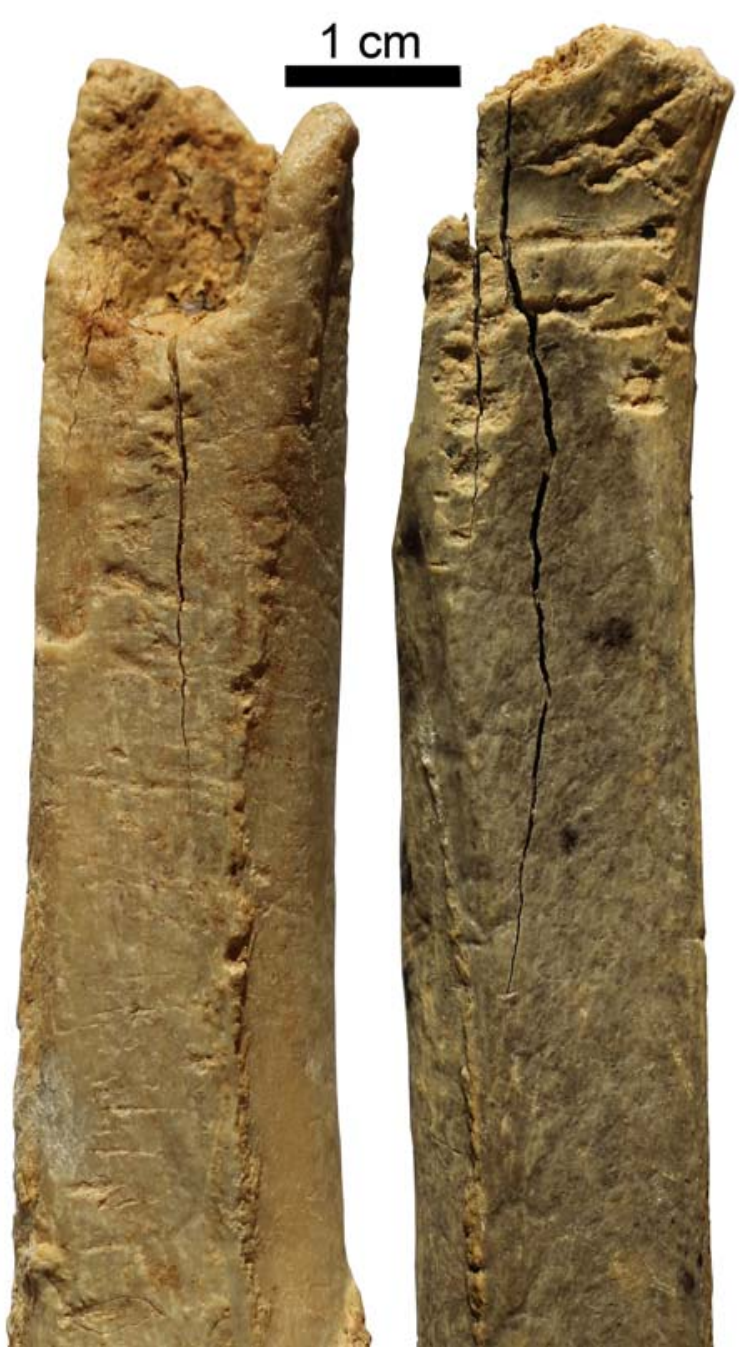

Fig. 13. Canis lupus, radius proximal fragments with signs of gnawing by large carnivores (presumably, by cup of hyenas), dorsal view (ZIN 37274-36, left and ZIN 37274-35, right).

Genus Cuon Hodgson, 1838

Cuon alpinus (Pallas, 1811)

In number of bone remains recovered in Geographical Society Cave, Cuon alpinus is noticeably inferior to Canis lupus. Ovodov (1977) counted 20 specimens of the red wolf; the examined material seems to confirm only 10 fossil remains.

There is the mandibular fragment (ZIN 37276) with $\mathrm{p} 4$ and $\mathrm{m} 1$ and alveoli of p2, p3 and $\mathrm{m} 2$ (Tab. 11, Fig. 14). By its height in front of $\mathrm{ml}$ (25.2 mm), ZIN 37276 corresponds to the largest fossil specimens of $C$. $a$. caucasicus from Kudaro 3 Cave in Caucasus (Baryshnikov, 2012); however, the greatest length of its $\mathrm{m} 1$ does not differ from that in the majority of $\mathrm{m} 1$ specimens from the Caucasian collection. The length of $\mathrm{p} 4$ $\mathrm{m} 1(33.8 \mathrm{~mm})$ is similar to maximum values of this dimension in the recent subspecies C. a. alpinus from the Russian Far East. 
Table 11. Measurements (mm) of mandibles of Cuon alpinus and Vulpes vulpes.

\begin{tabular}{|c|c|c|c|c|c|}
\hline \multirow{3}{*}{ Measurements } & \multicolumn{3}{|c|}{ C. alpinus } & \multicolumn{2}{|c|}{ V. vulpes } \\
\hline & \multicolumn{2}{|c|}{ Geographical Society Cave } & $\begin{array}{c}\text { Primorskii Territory, } \\
\text { recent }\end{array}$ & $\begin{array}{l}\text { Geographical } \\
\text { Societv Cave }\end{array}$ & $\begin{array}{c}\text { Primorskii } \\
\text { Territory recent }\end{array}$ \\
\hline & ZIN 37276 & ZIN 37277-6 & ZIN $18262,0^{7}$ & ZIN 37286 & ZIN $16706,0^{7}$ \\
\hline Lc1-m3, alv & & & & ca77 & 72.8 \\
\hline $\mathrm{Lp} 1-\mathrm{m} 3$ & & & & 67.6 & 59.9 \\
\hline Lp1-p4 & & & & 37.8 & 34.2 \\
\hline Lp4-m1 & 34.0 & & 34.5 & alv28.7 & 25.8 \\
\hline Lm1-m2 & & & & 26.3 & 23.5 \\
\hline Height behind $\mathrm{m} 1$ & 26.1 & & 23.8 & 17.4 & 14.5 \\
\hline Height behind $\mathrm{p} 2$ & 23.6 & & 20.4 & 14.9 & 12.5 \\
\hline \multicolumn{6}{|l|}{ Teeth } \\
\hline Lp4 & 13.9 & & 13.7 & & \\
\hline Wp4 & 6.2 & & 6.5 & & \\
\hline Lm1 & 21.6 & 22.5 & 22.9 & 18.3 & 15.8 \\
\hline Ltldm1 & 5.5 & 6.5 & 5.9 & 6.0 & 5.0 \\
\hline Wm1 & 9.1 & 10.9 & 8.5 & 7.0 & 6.4 \\
\hline Lm2 & & & & 8.3 & 7.2 \\
\hline $\mathrm{Wm} 2$ & & & & 6.1 & 5.5 \\
\hline
\end{tabular}

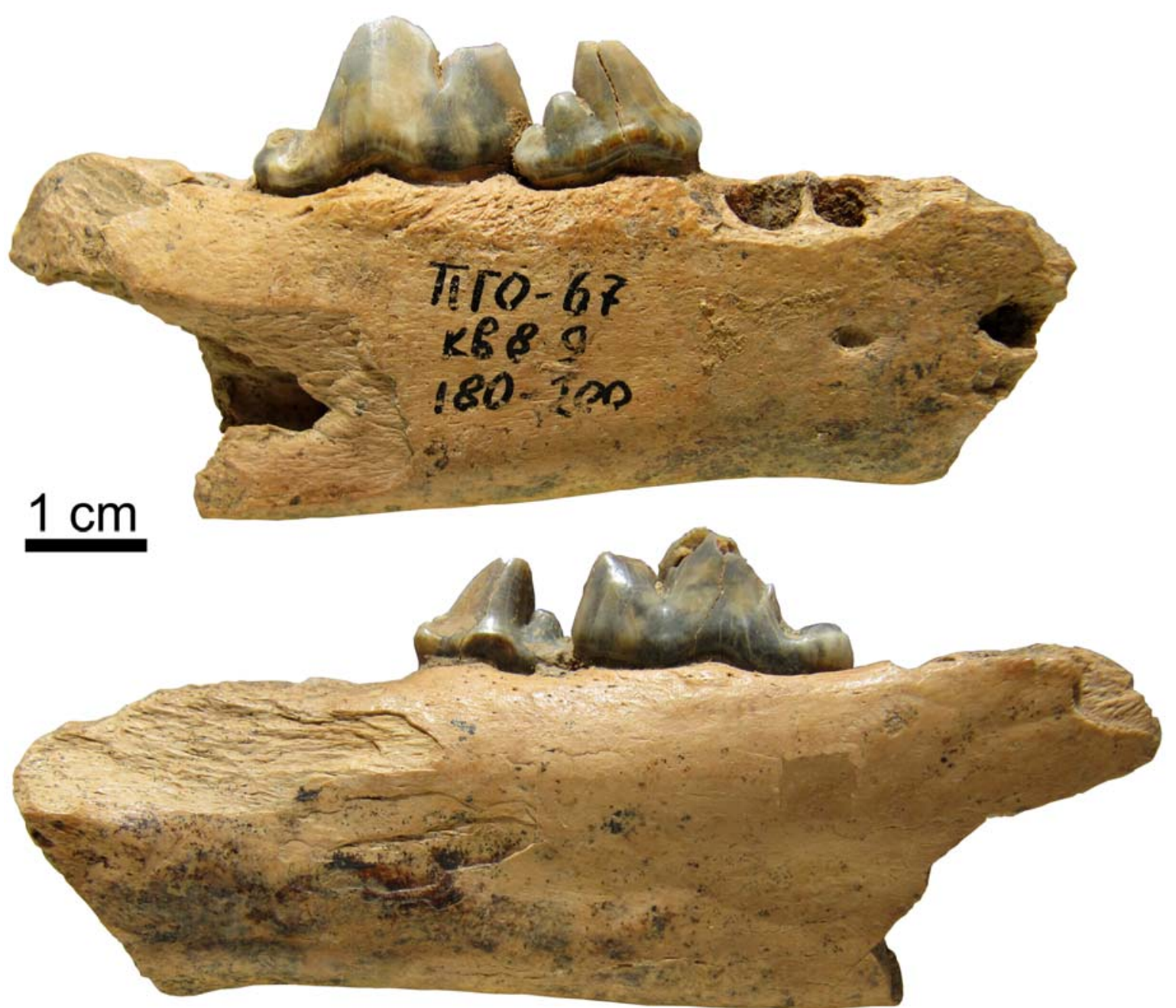

Fig. 14. Cuon alpinus, right mandible (ZIN 37276) from Geographical Society Cave, buccal (A) and lingual (B) views. 


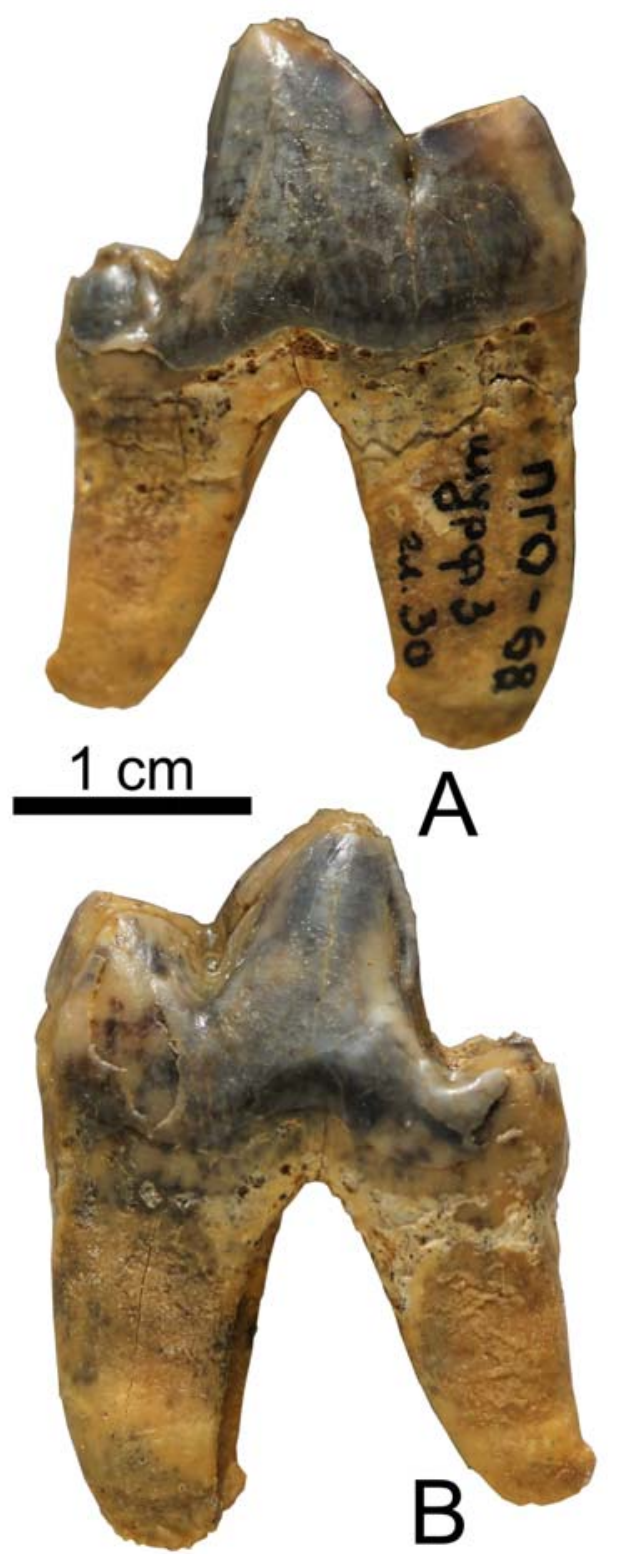

Fig. 15. Cuon alpinus, right lower molar m1 (ZIN 37277-6) from Geographical Society Cave, buccal (A) and lingual (B) views.
The lower premolar $\mathrm{p} 4$ is characterized by the high protoconid with a large accessory cusp at its base. A still smaller cuspid is observed behind the accessory cusp.

The lower carnassial m1 may be narrow (ZIN 37276) or somewhat widened (ZIN 37277-6) and exceed teeth of $C$. a. caucasicus in the width. By the length it corresponds to this tooth of the fossil C. alpinus from Upper Cave at Zhoukoudian in China (Pei, 1940). In both $\mathrm{m} 1$ specimens from Geographical Society Cave, metaconid is practically absent (Fig. 14, 15). The talonid is short; the robust hypoconid is shifted to its middle part. An inconspicuous transverse crest is present on the lingual side of hypoconid in ZIN 37277-6. The anterior-internal part of talonid is shaped as a small platform. Entoconid is not developed, but there is a rather low, serrated ridge along the talonid lingual margin. The crown buccal side bears a weak cingulid.

Several postcranial bones were found. The metacarpal 5 (ZIN 37277-15) is smaller and more slender as compared to the same bone of C. lupus (Tab. 12). In addition, there is a marked crest on the plantar side, which is inconspicuously defined in C. lupus (Fig. 16). The tibia (ZIN 37277-8) reveals smaller size than tibias of $C$. lupus, corresponding to that of $C$. a. caucasicus (Baryshnikov, 2012). Both tali (ZIN 37277-12, 3727713) differ from the analogous bones of C. lupus by smaller size and by more pronouncedly backwards extended extremity of upper trochlea in the area of its sulcus (Fig. 17).

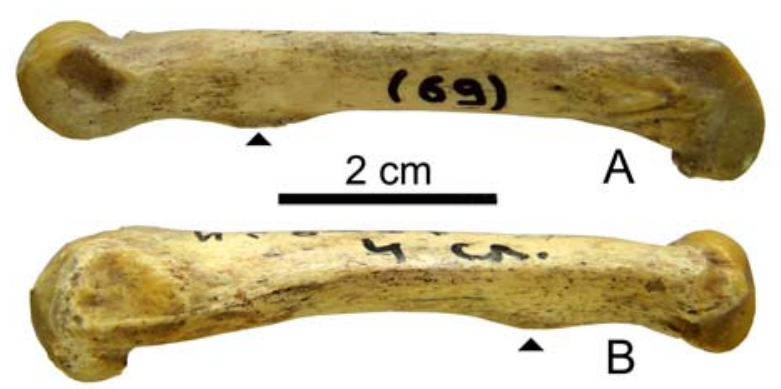

Fig. 16. Cuon alpinus, right metacarpal 5 (ZIN 37277-15) from Geographical Society Cave, medial (A) and lateral (B) views. Plantar ridge mark of up arrow.

Table 12. Measurements ( $\mathrm{mm}$ ) of hind limb bones of Late Pleistocene Cuon alpinus from Geographical Society Cave.

\begin{tabular}{|c|c|c|c|c|c|c|c|c|c|c|}
\hline Bone & $\begin{array}{l}\text { Museum } \\
\text { number }\end{array}$ & GL & $\mathrm{Bp}$ & $\mathrm{Bd}$ & SD & $\mathrm{Bd}$ & $\mathrm{Dd}$ & GLP & BG & GB \\
\hline \multicolumn{11}{|c|}{ Hind limb } \\
\hline Scapula & ZIN $37277-7$ & & & & & & & 33.6 & 21.6 & \\
\hline Radius & ZIN 37277-9 & & & & 16.6 & & & & & \\
\hline Mc5 & ZIN 37277-15 & 62.5 & 12.6 & 13.4 & 8.7 & 12.2 & 11.5 & & & \\
\hline \multicolumn{11}{|c|}{ Fore limb } \\
\hline Tibia & ZIN 37277-8 & & & & 15.2 & 27.7 & & & & \\
\hline \multirow{2}{*}{$\begin{array}{l}\text { Calcane } \\
\text { us }\end{array}$} & ZIN 37277-10 & 52.3 & & & & & & & & 21.7 \\
\hline & ZIN 37277-11 & 50.9 & & & & & & & & 22.7 \\
\hline \multirow{2}{*}{ Talus } & ZIN $37277-12$ & 31.3 & & & & & & & & 25.5 \\
\hline & ZIN 37277-13 & 30.1 & & & & & & & & 27.2 \\
\hline
\end{tabular}




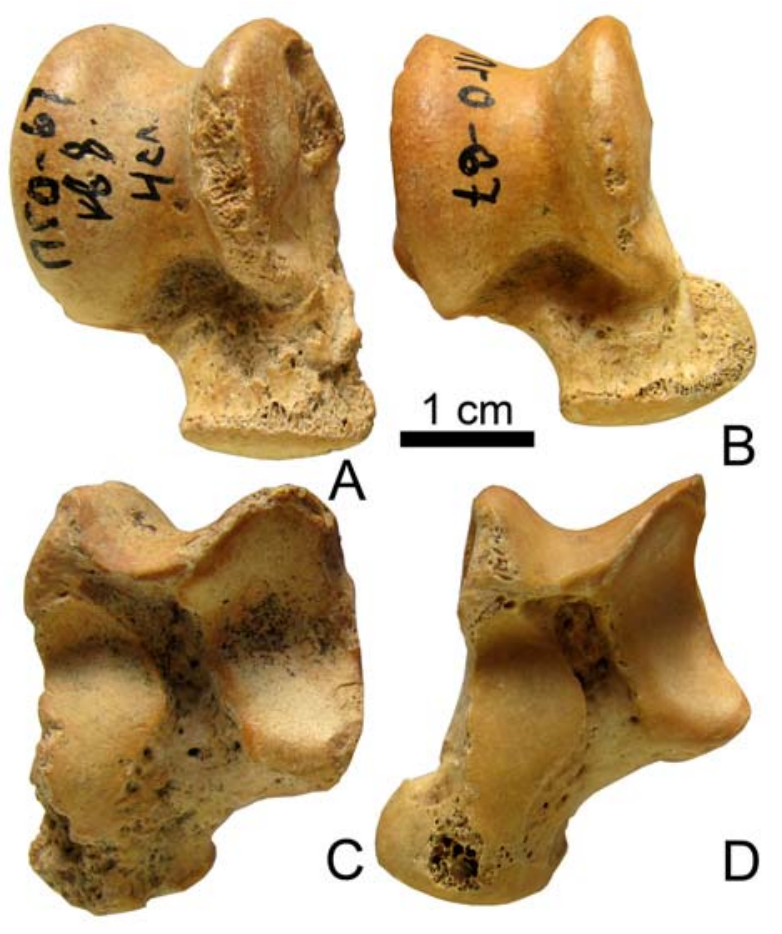

Fig. 17. Cuon alpinus, right tali from Geographical Society Cave, dorsal (A, B) and ventral (B, C) views. A, C - ZIN 37277-12; B, D - ZIN 37277-13.

Genus Vulpes Frisch, 1775

Vulpes vulpes (Linnaeus, 1758)

Ovodov (1977) referred 19 bones of the red fox to 4 individuals. The material examined by me comprises 17 fossil remains of $V$. vulpes.

The right mandible (ZIN 37286-1) is large (Tab. 11). Its height behind the $m 1$ as well as length of $m 1$ and $\mathrm{m} 2$ markedly exceed those of fossil mandibles from Kudaro caves in Caucasus (Baryshnikov, 2012) as well as mandibles of the recent $V$. vulpes dolichocrania Ognev, 1926 from Primorskii Territory.

The upper dentition is represented by the isolated canine (ZIN 37286-4), two premolars P3 (ZIN 372869, 37286-6), and the molar M2 (ZIN 37286-2). The canine $(\mathrm{L}=6.7 \mathrm{~mm}, \mathrm{~W}=4.5 \mathrm{~mm})$ corresponds by its size to canines of the recent $V$. vulpes. $\mathrm{P} 3$ is shaped ordinary of $V$. vulpes. Dimensions of both specimens $(\mathrm{L}=8.9$, 9.3, $\mathrm{W}=3.6,3.9, \mathrm{~mm}$, correspondingly, $n=2$ ) resemble those of the recent red fox. The M2 does not differ by its length $(6.2 \mathrm{~mm})$ and width $(8.9 \mathrm{~mm})$ from the teeth of the recent $V$. vulpes. The paracone is markedly higher and larger than metacone. A well-defined cingulum is extended at bases of both cusps. The protocone is rather small and distanced from the paracone. The metaconule is smaller and adjoined to the metacone. There is a minute accessory cusp between the protocone and metaconule, so as three cusps enclose lingually the talon basin. The cingulum forms a high and elongated elevation, hypocone, on the lingual margin of the crown. The tooth exhibits 3 roots.

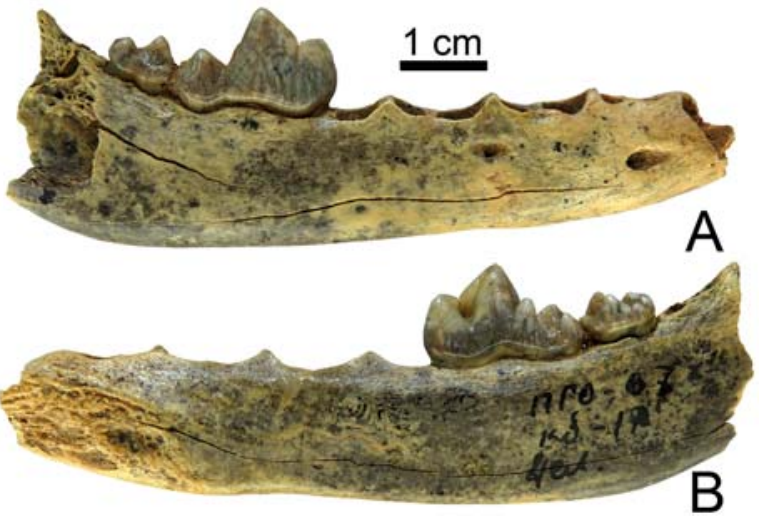

Fig. 18. Vulpes vulpes, right mandible fragment (ZIN 372861); buccal (A) and lingual (B) views.

The dimensions of the lower canine ZIN 37286-3 $(\mathrm{L}=8.6 \mathrm{~mm}, \mathrm{~W}=5.1 \mathrm{~mm}$ ) make it possible to assign it to a female. There are also two lower premolars p2 (ZIN 37286-5 and 37286-8). Their size ( $\mathrm{L}=8.7,8.9 \mathrm{~mm}$, $\mathrm{W}=3.8,3.8 \mathrm{~mm}$, respectively, $n=2$ ) is corresponds to that of the recent $V$. vulpes.

The tooth $\mathrm{m} 1$ from the mandible ZIN 37286-1 reveals a very high protoconid (Fig. 18). The metaconid is distinct and well separated from the posterior margin of protoconid. The talonid is characterized by the basin opened lingual. The hypoconid is robust and undivided. The entoconid is markedly smaller and lower and more pronouncedly shifted backwards with regard to hypoconid. Hypoconulid is not developed. The lower premolar m2 from specimen ZIN 37286-1 has a high protoconid and lower metaconid. Apices of both cusps lay on the same level in the lateral view. The depression (talonid basin) is placed behind these cusps, being surrounded by hypoconid, entoconid, and a miniature entoconulid. The crown has a cingulid visible in its anterior-external angle.

Bones of postcranial skeleton resemble by their size and morphology those of the recent $V$. vulpes (Tab. 13).

\section{Conclusions}

The analysis of paleontological collection from Geographical Society Cave ascertains the presence of the fossil remains of four canid species: Nyctereutes procyonoides, Canis lupus, Cuon alpinus, and Vulpes vulpes. These species occur in Primorskii Territory at present time (with exception for the recently extinct dhole). Therefore, the canid species diversity did not undergo changes in the southern part of the Russian Far East from the Late Pleistocene until now, i.e. over 40 thousands years.

Wolf (Canis lupus) was widely distributed in the Late Pleistocene in Siberia: from Altai and Sayan Mountains to the Arctic coast northwards. Red fox (Vulpes vulpes) was confined predominantly to the southern regions of Siberia, not migrating far northwards (Boesko- 
Table 13. Measurements ( $\mathrm{mm}$ ) of limb bones in Late Pleistocene Vulpes vulpes from Geographical Society Cave.

\begin{tabular}{|l|c|c|c|c|c|c|c|c|c|c|c|}
\hline \multicolumn{1}{|c|}{ Bone } & $\begin{array}{c}\text { Museum } \\
\text { number }\end{array}$ & GL & Bp & SD & Bd & SDO & DPA & BPC & SLC & GLP & BG \\
\hline \multicolumn{7}{|c|}{ Hind limb } \\
\hline Scapula & ZIN 37286-17 & & & & & & & & 19.0 & 20.7 & 12.8 \\
\hline Humerus & ZIN 37286-10 & & & 7.8 & 20.5 & & & & & & \\
\hline Ulna & ZIN 37286-15 & & & & & 14.1 & 17.2 & 9.5 & & & \\
\hline Radius & ZIN 37286-14 & & 12.9 & 8.9 & & & & & & & \\
\hline Mc4 & ZIN 37286-16 & 52.2 & 5.7 & 4.2 & 6.4 & & & & & & \\
\hline \multicolumn{7}{|c|}{ Fore limb } & & & & & \\
\hline \multirow{2}{*}{ Tibia } & ZIN 37286-12 & & 22.0 & 9.3 & & & & & & & \\
\hline
\end{tabular}

rov \& Baryshnikov, 2013). Dhole (Cuon alpinus), presumably, occurred in the southern mountain ranges of Southern Siberia; no finds of its dens or its cubs is known there in the historical time, which suggests only occasional occurences of these animals during their long migrations. The raccoon dog (Nyctereuites procyonoides) is recorded in the Late Pleistocene of Asiatic Russia only in the localities of Primorskii Territory. Therefore, the Late Pleistocene canid complex established on the basis of the collections from Geographical Society Cave involves hunters of mid-size ungulates (Canis lupus, Cuon alpinus), a hunter of rodents and birds (Vulpes vulpes), and a gatherer of smaller vertebrates and invertebrates (Nyctereutes procyonoides), which testifies the former abundance of fauna in the southern part of the Russian Far East.

A part of canid remains may have been accumulated in the Geographical Society Cave as a result of natural mortality of animals within the cave cavity. Nevertheless, the main source of bone accumulation appears to be a hunting activity of larger carnivores: hyena ( $\mathrm{Cro}$ cuta ultima) and large cats (Panthera tigris/ P. spelaea). Wolves and hyenas could also take a part in the modification of the bone assemblage in the cave. No traces of canid utilization by ancient people have been detected, suggesting the cave was used by hominins as a shorttermed shelter.

\section{Acknowledgements}

I am grateful to late Prof. N.K. Vereshchagin (Saint Petersburg) and Dr. N.D. Ovodov (Krasnoyarsk) who collected fossil bone material from Geographical Society Cave. I am grateful to Drs. G. Boeskorov and I. Belolubsky (Yakutsk, Russia), late Dr. N.L. Fomicheva (Kazan), Prof. A. Nadachowski (Krakow), Dr. J. Wagner (Prague), Prof. A. Lister and A. Currant (London), and T. Schlossleitner (Berlin). My wife, Svetlana Baryshnikova, contributed to the improvement of the manuscript. Prof. J. Hoffecker (Boulder, Colorado, USA) made several corrections. I am especially obliged to referees Dr. Marina Sotnikova (Moscow), Prof. Rafaelle Sardela (Roma), and Dr. Jan Wagner (Prague) for valuable comments to the manuscript. The study is finan- cially supported by the Russian Foundation for Basic Research, project no. 13-04-00081-a.

\section{References}

Baryshnikov G. 2012. Pleistocene Canidae (Mammalia, Carnivora) from the Paleolithic Kudaro caves in the Caucasus // Russian Journal of Theriology. Vol.11. No.2. P.77120.

Baryshnikov G. 2014. Late Pleistocene hyena Crocuta ultima ussurica (Mammalia, Carnivora, Hyaenidae) from the Paleolithic site in Geographical Society Cave in the Russian Far East // Proceeding of the Zoological Institute RAS. Vol.318. P.197-225.

Baryshnikov G., Mol D. \& Tikhonov A. 2010. Finding of the Late Pleistocene Carnivora in Taimyr Peninsula (Russia, Siberia) with paleoecological context // Russian Journal of Theriology. Vol.8. No.2. P.107-113.

Boeskorov G.G. \& Baryshnikov G.F. 2013. [Late Quaternary Carnivora of Yakutia]. Saint-Petersburg: Nauka. 199 p. [in Russian].

Driesch A., von den. 1976. A guide to the measurement of animal bones from archaeological sites // Peabody Museum Bulletin. Vol.1. P.1-136.

Kostoglod V.E. 1980. [Wolf] // Rastitel'nyi i Zhivotnyi Mir Sikhote-Alinskogo Zapovednika. Moscow: Nauka. P.228-230 [in Russian].

Kuzmin Y.V., Baryshnikov G.F., Timothy J., Orlova L.A. \& Plicht J. van der. 2001. Radiocarbon chronology of the Pleistocene fauna from Geographic Society Cave, Primorye (Russian Far East). Current Research in the Pleistocene. Vol.18. P.106-108.

Leonard J.A., Vila C., Fox-Dobbs K., Koch P.L., Wayne R.K. \& Van Valkenburgh B. 2007. Megafaunal extinctions and the disappearance of a specialized wolf ecomorph // Current Biology. Vol.17. P.1146-1150.

Ovodov N.D. 1977. [Late Quaternary fauna of mammals (Mammalia) of south of Ussiry region.] // Yudin B.S. (ed.). Fauna i Sistematika Pozvonochnykh Sibiri. Novosibirsk: Nauka. P.157-177. [in Russian].

Pei W.C. 1934. On the Carnivora from Locality 1 of Choukoutien // Palaeontologia Sinica. Ser.C. Vol.8. P.1-216.

Pei W.C. 1936. On the mammalian remains from Locality 3 at Choukoutien // Palaeontologia Sinica. Ser.C. Vol.7. No.5. P.1-108. 
Pei W.C. 1940. The Upper Cave Fauna of Choukoutien // Palaeontologia Sinica. Ser.C. Vol.10. P.1-84.

Rohland N., Pollack J.L., Nagel D., Beauval C., Airvaux J., Pääbo S. \& Hofreiter M. 2005. The population history of extant and extinct hyenas // Molecular Biology and Evolution. Vol.22. No.12. P.2435-2443.

Sala N., Arsuaga J.L. \& Haynes G. 2014. Taphonomic comparison of bone modifications caused by wild and captive wolves (Canis lupus) // Quaternary International. Vol.330. P.126-135.

Sardella R., Bertè D., Iurino D.A., Cherin M. \& Tagliacozzo A. 2014. The wolf from Grotta Romanelli (Apulia, Italy) and its implications in the evolutionary history of Canis lupus in the Late Pleistocene of Southern Italy // Quaternary International. Vol.328-329. P.179-195.

Stuart A.J. \& Lister A.M., 2014. New radiocarbon evidence on the extirpation of the spotted hyaena (Crocuta crocuta (Erxl.)) in northern Eurasia. Quaternary Science Reviews. Vol.96. P.108-116.
Tong H.-W., Hu N. \& Wang X.-M. 2012. New remains of Canis chihliensis (Mammalia, Carnivora) from Shanshenmiaozui, a Lower Pleistocene site in Yangyuan, Hebei // Vertebrate PalAsiatica. Vol.50. No.4. P.335-360.

Valova Z.G., Vasil'ev N.G., Zhivotchenko V.I., Makovkin L.I., Oliger T.I., Prisyazhniuk V.E., Prisyazhniuk N.P., Solomkina N.V., Khramtsov V.S. \& Shaldybin S.L. 1989. [Lazovsky Reserve]. Moscow: Agropromizdat. 206 p. [in Russian].

Vereshchagin N.K. 1971. [The cave lion and his history in Holarctic and in territory of USSR] // Trudy Zoologicheskogo Instituta AN SSSR. Vol.49. P.123-199 [in Russian with English summary].

Yudin V.G. 2013. [The Wolf of the Far East of Russia]. Vladivostok: Dalnauka. 412 p. [in Russian].

Yudin V.G. \& Yudina E.V. 2009. [The Tiger of the Far East of Russia]. Vladivostok: Dalnauka. 485 p. [in Russian].

Zhang Zh.-Q. 2001. Fossil mammals of early Pleistocene from Ningyang, Shandong Province // Vertebrate PalAsiatica. Vol.39. No.2. P.139-150. 